\title{
Conjugated Polymer Blends for Organic Thermoelectrics
}

Guangzheng Zuo, Hassan Abdalla and Martijn Kemerink

The self-archived postprint version of this journal article is available at Linköping University Institutional Repository (DiVA):

http://urn.kb.se/resolve?urn=urn:nbn:se:liu:diva-162544

N.B.: When citing this work, cite the original publication.

Zuo, G., Abdalla, H., Kemerink, M., (2019), Conjugated Polymer Blends for Organic Thermoelectrics, ADVANCED ELECTRONIC MATERIALS, 5(11), 1800821. https://doi.org/10.1002/aelm.201800821

Original publication available at:

https://doi.org/10.1002/aelm.201800821

Copyright: Wiley (12 months)

http://eu.wiley.com/WileyCDA/ 


\section{WILEY-VCH}

DOI: 10.1002/ ((please add manuscript number))

Article type: Progress Report

\section{Conjugated Polymer Blends for Organic Thermoelectrics}

Guangzheng Zuo, Hassan Abdalla and Martijn Kemerink*

((Optional Dedication))

Dr. G. Zuo, Dr. H. Abdalla, Prof. M. Kemerink

Complex Materials and Devices, Department of Physics, Chemistry and Biology, Linköping

University, 58183 Linköping, Sweden

E-mail: martijn.kemerink@liu.se

Keywords: Seebeck coefficient, conjugated polymers, blending, organic thermoelectrics, charge transport models

\section{Abstract:}

A major attraction of organic conjugated semiconductors is that materials with new, emergent functionality can be designed and made by simple blending, as is extensively used in e.g. bulk heterojunction organic solar cells. Here, we critically review doped blends based on organic semiconductors (OSC) for thermoelectric applications. Several experimental strategies to improve thermoelectric performance, measured in terms of power factor (PF) or figure-ofmerit ZT, have been demonstrated in recent literature. Specifically, density-of-states design in blends of 2 OSCs can be used to obtain electronic Seebeck coefficients up to $\sim 2000 \mu \mathrm{V} / \mathrm{K}$. Alternatively, blending with (high-dielectric constant) insulating polymers can improve doping efficiency and thereby conductivity, as well as induce more favorable morphologies that improve conductivity while hardly affecting thermopower. In the PEDOT:PSS blend system, processing schemes to either improve conductivity via morphology or via (partial) removal of the electronically isolating PSS, or both, have been demonstrated. Although a range of experiments have at least quasi-quantitatively been explained by analytical or numerical models, a comprehensive model for organic thermoelectrics is lacking so far. 


\section{WILEY-VCH}

\section{Introduction}

The study of semiconducting organic polymers for their thermoelectric properties has in recent years become an activity of substantial size, both in terms of number of active researchers and of number of papers published. In view of the earlier, much smaller attention for organic thermoelectric polymers and especially the modest performances achieved before this decennium, this interest was not upfront evident. ${ }^{[1]}$ A major attraction of (polymer) organic semiconductors (OSC) is their thermal conductivity that, due to their structurally disordered nature, can be as low as $\kappa \sim 0.2 \mathrm{~W} \mathrm{~m}^{-1} \mathrm{~K}^{-1},{ }^{[2,3]}$ whereas pure silicon has $\kappa \sim 150 \mathrm{~W} \mathrm{~m}^{-1} \mathrm{~K}^{-1},{ }^{[4]}$ and the best reported $\mathrm{Bi}_{2} \mathrm{Te}_{3}$ has $\sim 1.5 \mathrm{~W} \mathrm{~m}^{-1} \mathrm{~K}^{-1} \cdot{ }^{[5]}$ Nevertheless, earlier reported OSC failed to show a combination of conductivity $\sigma$ and thermopower (or Seebeck coefficient) $S$ that led to application-relevant power factors $P F=S^{2} \sigma$ and/or figures of merit $Z T=P F / \kappa T$ that approach unity. In 2011 Bubnova et al. reported $Z T \approx 0.25$ for the p-type OSC PEDOT:Tos by optimizing the oxidation level, which still represents the state-of-theart. ${ }^{[6]}$ Later, Kim et al. reported ZT $\approx 0.42$ for the related PEDOT:PSS system, but this work has been disputed because of the used measurement geometry. ${ }^{[7-9]}$

For inorganic materials it is well known that improving ZT is a far from trivial task due to the fact that the constituent parameters $S, \sigma$ and $\kappa$ are typically counter-indicated, and strategies to improve one tend to backfire by reducing the others. ${ }^{[10]}$ For organic materials similar relations are, certainly at the formal level, less well established. Nevertheless, the empirically established inverse (power law) relation between $S$ and $\sigma$, and linear proportionality between $\sigma$ and $\kappa$ strongly suggest that a similar trade-off exists for OSC. ${ }^{[11,12]}$ In terms of rational optimization of polymer OSC for thermoelectric applications, especially the empirical relation $S \propto \sigma^{-1 / 4}$ implies an important design rule as it translates into $P F \propto \sigma^{1 / 2}$ : the positive effects of increasing (electrical) conductivity outweigh the associated negative effects of decreasing thermopower, at least as long as the thermal conductivity is more or less constant. 


\section{WILEY-VCH}

In other words, charge carrier densities, and therefore doping levels, should be maximized while minimizing any effects that deteriorate the mobility. Indeed, significant research efforts are currently put in increasing doping levels and doping efficiencies without compromising the microstructure of the pristine material. ${ }^{[1,13-16]}$ At the same time, increasing the doping level is only one out of many strategies followed to increase the thermoelectric performance of OSC. For instance, encouraging results have been obtained with improving doping efficiency by increasing the dielectric constant of the host matrix. ${ }^{[17-19]}$ Alternatively, targeted changes in the structure of either the backbone or the side chains of the conjugated polymer have been reported to give improved performance. ${ }^{[20-23]}$ Unfortunately, these strategies can sometimes be hard to translate one-on-one to other materials, somewhat limiting their use as general design rules.

Another approach towards better organic thermoelectric materials is by using the possibilities offered by blending, that is to create materials with desired properties that are not just the sum of their constituents, by 'simply' mixing materials. The possibility to blend conjugated semiconductors enables, for instance, the fabrication of organic bulk heterojunction solar cells. This Progress Report aims to give an overview of selected results for conjugated polymer blends for thermoelectric applications, as well as their formal interpretation. Specifically, we will discuss blends of conjugated polymers with either another conjugated polymer, or with a non-conjugated polymer. The latter can be either electrically inert, like polystyrene, or ionically conductive like polystyrene-sulfonate (PSS). In the systems discussed, the charge and energy transport take place entirely through the conjugated polymer(s). As a complementary approach, hybrid blends of conjugated polymers with for example carbon nanotubes, graphene or inorganic nanoparticles have shown promising results in terms of power factor and figure of merit. ${ }^{[24-26]}$ In these compounds both the host and guest phases are involved in the charge transport. Since the transport in the guest phase requires a different formalism (for band transport) than that in conjugated polymers on which we focus 


\section{WILEY-VCH}

here, these compounds fall outside the scope of this Report and will only be mentioned in passing.

\section{Fundamentals of organic thermoelectrics}

Before turning to experimental results for the thermoelectric properties of conjugated polymer blends, we shall first shortly review some formal frameworks that have been used to rationalize such results. Despite the inherent simplifications and limitations of current models, they often give surprisingly accurate descriptions of key parameters of neat and blended organic thermoelectrics and as such are a starting point for establishing a, so far missing, comprehensive, quantitative framework for organic thermoelectrics.

Two factors will play a key role in any quantitative description of charge and energy transport in any organic thermoelectric: the density of states (DOS) and the morphology. The DOS of intrinsic OSC is typically described as a Gaussian with a width around 50-120 meV. To achieve any relevant bulk conductivity, the intrinsic material will have to be doped, which means there must be counter charges, typically in the form of quasi-static ions, to the free electrons or holes that carry current and energy. The Coulomb potential of these ions will unavoidably induce a deep tail in the DOS of exponential shape, as was shown by Silver et al. ${ }^{[27]}$ The negative effect of this undesired DOS modification has been studied theoretically ${ }^{[28-30]}$ and experimentally. ${ }^{[31-35]}$ In contrast to the dopant-induced case, the DOS changes that arise from blending with a second conjugated material are intentional and can be used to enhance both $S$ and PF in a rational manner, as will be discussed in section 3.1. Finally, both processing and blending with non-ionic, non-conjugated materials may be expected to also lead to DOS changes, but such effects have, to our knowledge, not been studied explicitly. Incorporating morphology in quantitative models for organic thermoelectrics has so far hardly happened. In view of the experimentally established importance of for instance the position 


\section{WILEY-VCH}

and orientation of the dopant molecule with respect to the conjugated backbone, ${ }^{[36-38]}$ and the degree of crystallinity of the $\operatorname{OSC}^{[13-15]}$, this is a matter of some urgency. In section 3.2 a kinetic monte Carlo model that, in a simplified manner, accounts for phase separation in thermoelectric blends will be discussed.

In the remainder of this section we will review two hopping models. In both cases we will assume integer charge transfer (ICT) to be the dominant doping mechanism, i.e. hybridization between the electronic states of the dopant and the semiconductor(s), leading to charge transfer complex (CTC) formation, will be ignored. ${ }^{[38]}$ Nevertheless, most mechanisms outlined below can be expected to hold, at least qualitatively, also for CTC, albeit with different energetics due to the stabilization of the (partially) transferred charge in the hybrid CTC state. In addition, a generic transport edge model will be introduced for comparison.

\subsection{Analytical models for disordered organic thermoelectrics}

The charge transport mechanism in structurally disordered OSC is typically described as a thermally activated tunneling, or hopping, process. The hopping rate from an initial state $i$ with energy $E_{\mathrm{i}}$ to a final state $f$ with energy $E_{f}$ can be described by the Miller-Abrahams expression $^{[39]}$, which is based on a phonon-assisted tunneling mechanism, as

$v_{i f}=\left\{\begin{array}{c}v_{0} \exp \left(-2 \alpha r_{i f}\right) \exp \left(\frac{-E_{f}-E_{i}}{k_{B} T}\right)(\Delta E>0) \\ v_{0} \exp \left(-2 \alpha r_{i f}\right)(\Delta E \leq 0)\end{array}\right.$,

where $v_{0}$ is the carrier attempt-to-hop frequency, generally understood to be related to some phonon frequency in the material, $r_{i f}$ is the distance between the localized sites, $k_{\mathrm{B}}$ is the Boltzmann constant, $T$ is the temperature and $\alpha$ describes the decay length of the localized wavefunctions. For intrinsic materials, the energy distribution of the localized sites through which hopping takes place is assumed to be Gaussian:

$g_{0}\left(E_{i}\right)=\frac{N_{0}}{\sqrt{2 \pi \sigma_{D O S}^{2}}} \exp \left(\frac{-\left(E_{i}-E_{0}\right)^{2}}{2 \sigma_{D O S}^{2}}\right)$ 


\section{WILEY-VCH}

where $E_{\mathrm{i}}$ is the single particle energy on site $i, E_{0}$ and $\sigma_{D O S}$ are the mean and the standard deviation (or simply the energetic disorder) of the Gaussian DOS, respectively, and $N_{0}$ is the total site density.

To calculate macroscopic observables like conductivity or thermopower, it is common to use arguments from percolation theory. In a nutshell, the conductivity of the infinite percolating network, i.e. the sample conductivity, is assumed to be characterized by a 'critical' or 'characteristic' hop. Below we shall introduce a specific incarnation of such a model as proposed by Zuo et al., following the work by Martens et al. ${ }^{[11,40]}$ This model can deal with arbitrary DOS shapes, which is crucial in the current context, and has previously been shown to give an accurate description of the carrier density dependence of the mobility in an (unperturbed) Gaussian DOS. ${ }^{[41]}$ Moreover, it can easily be rendered from a variable range hopping $(\mathrm{VRH})$ model to a strictly nearest neighbor hopping $(\mathrm{nnH})$ model. For a more general introduction to percolation and hopping models, we refer to the available literature. ${ }^{[41-45]}$

\subsubsection{Conductivity model}

The conductivity in the Mott-Martens model is calculated from a Miller-Abrahams-type expression as (c.f. Eq. 1):

$\sigma=\sigma_{0} \exp \left(-2 \alpha R^{*}-\frac{E^{*}-E_{F}}{k_{B} T}\right)$

where $\sigma_{0}$ is a conductivity prefactor. The characteristic hop is assumed to take place over a distance $R^{*}$ and to go from the Fermi energy $E_{F}$ to an energy $E^{*}$. Note that in a Gaussian DOS at low charge densities, which are irrelevant to thermoelectric applications, the Fermi energy should be replaced by the equilibrium energy $E_{\infty}=-\sigma_{D O S}^{2} / k T$ as lower characteristic energy, leading to the well-known expression for the mobility $\mu(T) \propto \exp \left(-\left(C \sigma_{D O S} / k_{B} T\right)^{2}\right)$. Further, the characteristic hopping distance and energy difference $R^{*}$ and the critical or transport energy $E^{*}$ are connected to each other through the density of states $g(E)$ :

$B_{C}=\frac{4}{3} \pi R^{* 3} \int_{E_{F}}^{E^{*}} g(E) d E$ 


\section{WILEY-VCH}

where $B_{C} \approx 2.8$ is the critical number of bonds on the percolating network. For VRH systems, the conductivity is obtained by maximizing Eq. 3 under the condition Eq. 4 which gives rise to the well-known temperature-dependent tradeoff between 'long' (large $R^{*}$, low $T$ ) and 'high' hops $\left(\operatorname{large} E^{*}\right.$, high $T$ ). For nnH one has $R^{*}=a_{N N}$, with $a_{N N}=N_{0}^{-1 / 3}$ the nearest neighbor distance, so Eq. 4 becomes an explicit expression for $E^{*}$. Based on Cottaar et al., Zuo et al. assumed that $E^{*}$ from Eq. 4 could simply be replaced by a constant critical energy $E^{*}=E_{\text {crit }}=-0.491 \sigma_{D O S}$ that sits below the center of the DOS. ${ }^{[31,46]}$ For the ion-induced non-Gaussian DOS derived below, $E^{*}$ was calculated with respect to the DOS maximum. The conductivity prefactor $\sigma_{0}$ in Eq. 3 is calculated as

$\sigma_{0}=n \mu_{0}=N_{0} \frac{a_{t y p}^{2} v_{0}}{p k_{B} T}$

Here, $n$ is the charge carrier density that is set equal to the doping concentration $N_{d}-$ i.e. full integer charge transfer is assumed as mentioned above. Further, $a_{t y p}$ is the typical hopping distance that equals $a_{N N}$ for $\mathrm{nnH}$ and $R^{*}$ for VRH. Setting the constant $p=6$ worked well for $\mathrm{nnH}$ whereas $p \approx 1$ was used previously for $\mathrm{VRH} \cdot{ }^{[31,47]}$
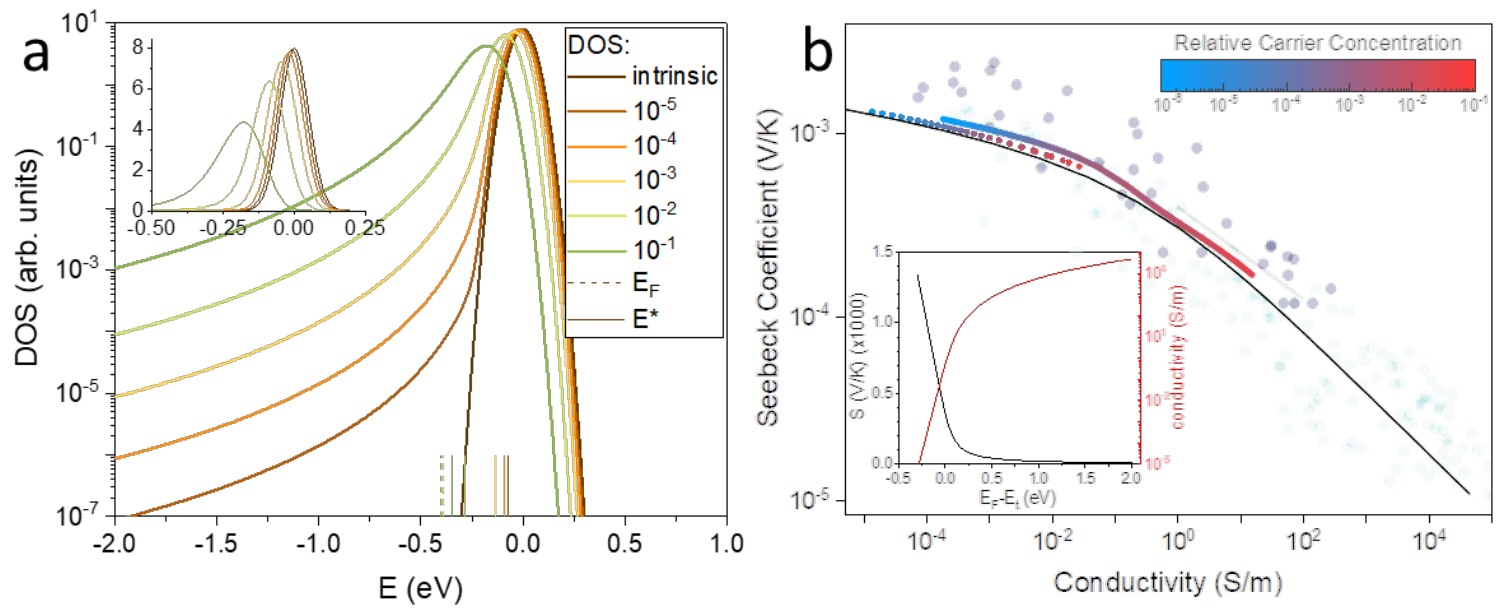

Figure 1. (a) Gaussian DOS $\left(\sigma_{D O S}=50 \mathrm{meV}\right.$ ) of an intrinsic OSC and with (relative) doping concentrations as indicated in the legend. Thin dashed and solid lines indicate $E_{F}$ and $E^{*}$, respectively. Inset: same data on linear scale. (b) Experimental thermopower vs. conductivity data from Ref. ${ }^{[11]}$ (green symbols) and own experiments (gray symbols). The gray straight line indicates the empirical $-1 / 4$ powerlaw relationship. Colored lines are model fits to the $\mathrm{nnH}$ (dotted) and VRH (solid) hopping model Eqs. 3-10; the thin solid line is the transport 


\section{WILEY-VCH}

edge model with $s=3$ and $\sigma_{E_{0}}(T)=0.1 \mathrm{~S} / \mathrm{m}$. The inset shows the underlying $S$ and $\sigma \mathrm{vs} E_{F}-$ $E_{t}$ curves in the transport edge model. Other parameters used: $T=300 \mathrm{~K}, a_{N N}=1.8 \mathrm{~nm}$, $\varepsilon_{r}=3.6, \alpha=0.5 \times 10^{9} \mathrm{~m}^{-1}$ and $\Delta E=0$. Panel $\mathrm{b}$ adapted with permission from Ref. ${ }^{[48]}$. Copyright 2017, APS.

\subsubsection{Ion perturbation of DOS}

Using computer simulations, Silver et al. have shown that an exponential tail of localized states can be induced by the long range electrostatic (Coulomb) potential due to a distribution of randomly positioned charges. ${ }^{[27]}$ Interestingly, the exponent depends upon the material's dielectric constant $\varepsilon_{r}$ and the site density and hardly on the charge density, and will therefore vary relatively little across different OSC. Later, Arkhipov et al. developed an analytical model for the ion-perturbed DOS of a doped semiconductor as ${ }^{[28]}$

$g(E)=A \int_{-\infty}^{0} \frac{d E_{C}}{E_{C}^{4}} \exp \left(\frac{A}{3 E_{C}^{3}}\right) g_{0}\left(E-E_{C}\right)$

with $A=\frac{4 \pi q^{6} N_{d}}{\left(4 \pi \varepsilon_{0} \varepsilon_{r}\right)^{3}}$ and $E_{C}$ the Coulomb interaction $E_{C}(r)=-q^{2} / 4 \pi \varepsilon_{0} \varepsilon_{r} r$. Zuo et al extended Eq. 6 to account for energy level differences between the dopant and the semiconductor, $\Delta E=E_{d}-E_{0}$ with $E_{d}$ a relevant energy level of the dopant. Defining $E_{1}=$ $E_{C}\left(N_{i}^{-1 / 3}\right)$ as the Coulomb energy one lattice constant away from the ionized dopant, $g(E)$ was written as ${ }^{[49]}$

$$
\begin{aligned}
& g(E)=\left(1-\frac{4 \pi N_{d}}{3 N_{i}}\right) \frac{g_{1}(E)}{\int_{-\infty}^{0} d E g_{1}(E)}+\frac{4 \pi N_{d}}{3 N_{i}} \frac{g_{2}(E)}{\int_{-\infty}^{0} d E g_{2}(E)} \\
& g_{1}(E)=A \int_{E_{1}}^{0} \frac{d E_{C}}{E_{C}^{4}} \exp \left(\frac{A}{3 E_{C}^{3}}\right) g_{0}\left(E-E_{C}\right) \\
& g_{2}(E)=A \int_{-\infty}^{E_{1}} \frac{d E_{c}}{E_{C}^{4}} \exp \left(\frac{A}{3 E_{C}^{3}}\right) g_{0}\left(E-\Delta E-E_{C}\right)
\end{aligned}
$$

Note that the corresponding equation in the original paper Ref. ${ }^{[31]}$ was wrong; in the actual calculations the correct expressions were used. Figure 1a shows a representative plot of a Gaussian DOS broadened by Coulombic interactions with static, randomly distributed ions as described by Eq. 7. For higher relative doping concentrations $c=N_{d} / N_{0}$ both $E_{F}$ and $E^{*}$ sit 


\section{WILEY-VCH}

in the ion-induced tail, implying that charge and energy transport become independent of the original DOS. Dopant-induced tail states and the crucial role of the parameter $\Delta E$ were further studied and experimentally established by Gaul et al. using a combination of DFT calculations and photoelectron spectroscopy. ${ }^{[50]}$

Two, potentially major, shortcomings of Eqs. 6 and 7 should be mentioned. First, it was pointed out by Arkhipov et al. that the spatial correlations that result from the slowly varying Coulomb potential are ignored when using $g(E)$ from Eq. 6 or 7 as input for the transport model Eqs. 3-5. ${ }^{[28]}$ Interestingly, Zuo et al. showed by explicit comparison to kinetic Monte Carlo (kMC, vide infra) that the proposed remedy is inaccurate, whereas the bare model Eq. 7 provided good agreement with the kMC simulations. Second, in deriving Eq. 6 an unscreened Coulomb interaction was used, whereas in an actual doped OSC the mobile counter charges, i.e. the electrons or holes, will screen $E_{C}(r)$. Both effects are automatically included in the $\mathrm{kMC}$ model discussed below in section 2.2 where this is discussed further.

\subsubsection{Thermopower}

Following the intuitive derivation by Fritzsche, the Seebeck coefficient of 'any' (semi)conductor can be calculated from ${ }^{[51]}$

$S=\frac{-k_{B}}{q} \int d E \frac{E-E_{F}}{k_{B} T}\left(\frac{-\partial f}{\partial E}\right) \frac{\sigma(E)}{\sigma}$

where $\sigma(E)$ is the (not measurable) conductivity distribution function that is related to the (measurable) device conductivity $\sigma$ as

$$
\sigma=\int d E\left(\frac{-\partial f}{\partial E}\right) \sigma(E)
$$

Note that in the original work of Fritzsche the derivative of the Fermi-Dirac distribution function $-\partial f / \partial E$ has been integrated in $\sigma(E)$. Under the assumption that $\sigma(E)$ is narrowly centered around the transport energy $E^{*},{ }^{[52]} \mathrm{Eq} .8$ can be further simplified to

$S \cong-\frac{E^{*}-E_{F}}{q T}$ 


\section{WILEY-VCH}

The latter simplification is not strictly necessary and analytical expressions for $\sigma(E)$ have been derived by for instance Schmechel and Ihnatsenka et al. ${ }^{[53,54]}$

Figure $1 \mathrm{~b}$ compares the hopping model outlined above to a wide range of literature data for doped polymers, excluding the PEDOT family of materials. As extensively discussed in earlier work Ref. ${ }^{[48]}$, the VRH version of the model gives an excellent description of the thermoelectric properties of doped OSC and explains the empirical power law relation $S \propto$ $\sigma^{-1 / 4}$, up to conductivities $\sim 10^{2} \mathrm{~S} / \mathrm{m}$; extending the curve to higher $\sigma$ and lower $\mathrm{S}$ is hardly possible with realistic parameters. Especially further increasing the doping level, that already runs up to $c=0.3$ in Figure 1b, seems unrealistic.

\subsubsection{Transport edge model}

In a recent paper, Kang and Snyder proposed a generic model for charge transport in conducting, i.e. doped, polymers. ${ }^{[55]}$ Rather than starting from a specific transport mechanism, the authors propose a generic expression for the conductivity distribution function as

$\sigma_{E}(E, T)=\left\{\begin{array}{c}\sigma_{E_{0}}(T) \times\left(\frac{E-E_{t}}{k_{B} T}\right)^{S} \quad\left(E>E_{t}\right) \\ 0 \quad\left(E \leq E_{t}\right)\end{array}\right.$.

Here, $E_{t}$ is the so-called transport edge and $\sigma_{E_{0}}(T)$ the transport coefficient. A value of $s=3$ for the exponent was found to give a good fit to experimental data for a wide range of doped polymers. Although the authors relate their model to a number of known transport models, a firm connection between Eq. 11 and specific modes of charge transport has not yet been made. As such, the physical meaning of the parameters in Eq. 11 remains somewhat unclear. A fit to the collected experimental data in Figure $1 \mathrm{~b}$ using the transport edge model outlined above is shown as thin solid black line. From a phenomenological perspective, the transport edge and variable range hopping models are, within typical experimental resolution, indistinguishable. The slope change in the former occurs when the Fermi energy moves beyond the transport edge, see the inset of Figure 1b. In order to cover the full experimentally observed conductivity range up to $\sim 10^{5} \mathrm{~S} / \mathrm{m}, E_{F}$ has to move $\sim 2 \mathrm{eV}$ beyond $E_{t}$. This is hard to 


\section{WILEY-VCH}

reconcile with the DOS shapes plotted in Figure 1a as well as with the results in Ref. ${ }^{[50]}$, and one can wonder how a DOS that can accommodate such a large shift in $E_{F}$ would have to look. In light of the problems noted for both the hopping model and the transport edge model, there is need for an extended theory to explain the experimentally observed thermoelectric behavior in the, practically most relevant, high-conductivity, low-thermopower regime.

\subsection{Kinetic Monte Carlo model for disordered organic thermoelectrics}

Several shortcomings of the analytical models discussed in the previous section 2.1 can, at the cost of increased computational demands, be overcome using quasi-atomistic kinetic Monte Carlo (kMC) models. In particular, kMC can relatively straightforwardly account for differences in morphology of phase separated systems and does automatically account (if properly set up) for correlations in the energy landscape due to the (screened) long-range Coulomb interaction between mobile charges and static ions as well as between mobile charges amongst themselves.

\subsubsection{Model description}

When considering nearest neighbor hopping, the used kMC models work on a simple cubic grid with lattice constant $a_{N N}=N_{0}^{-1 / 3}$; for variable range hopping, a random lattice of the same average site density is used. As for the analytical model in section 2.1, we use MillerAbrahams hopping rates (Eq. 1). Site energies $E_{0, i}$ are drawn from a Gaussian DOS (Eq. 2) and corrected for the interaction with all charges in the simulation box, i.e. other mobile charges and static dopant ions, viz.

$E_{i}=E_{0, i}+\sum E_{C}^{j}$

where the sum runs over all charges $j$ in the system but excludes on-site self-interaction. Periodic boundary conditions for motion and Coulomb interaction are used. To avoid divergences at zero separation between different particles, the Coulomb interaction is cut off 


\section{WILEY-VCH}

at a distance $r_{\min }$ such that $E_{C}=-q / 4 \pi \varepsilon_{0} \varepsilon_{r} r_{\min }=0.5 \mathrm{eV}$. In addition, relative site energies can be shifted by an externally applied electric field $F$.

Kinetic Monte Carlo models work by calculating, for a given configuration, all relevant site energies and from that all possible hopping rates. The waiting time before an event, i.e. a charge hop, occurs is then calculated as $\tau=-\ln (r) / \Sigma_{v}$ where $r$ is a random number drawn from a homogeneous distribution between 0 and 1 and $\Sigma_{v}$ is the sum of the rates of all possible events. The event that occurs after $\tau$ is selected randomly, using the rates of all possible events as weight factors. In principle, in presence of long-range Coulomb interactions between the mobile particles, energies and rates should be recalculated after each event. Significant calculation time reductions can be achieved by only recalculating these for the moving particle. The validity of this 'first reaction' scheme for thermoelectric simulations of OSC is unknown and full updates after each event will be used unless stated otherwise. The thermopower is calculated numerically exact from Eq. 8. Integrating $-\partial f / \partial E$ in $\sigma(E)$ and multiplying numerator and denominator by the applied electric field $F$, using $\sigma(E) F=$ $j(E)$, we get

$S=\frac{-k_{B}}{q} \int d E \frac{E-E_{F}}{k_{B} T} \frac{j(E)}{j}=\frac{-1}{q T}\left(\int d E E \frac{j(E)}{j}-E_{F}\right)$

In steady state, all currents are constant and can be integrated over time to obtain

$S=\frac{1}{q T}\left[\frac{\int_{0}^{t} \int E j(E) d E d t}{\int_{0}^{t} \int j(E) d E d t}-E_{F}\right]$

The double integrals reflect the total amount of charge that passes through a unit cross section in a given time $t$, in the numerator weighted by the charge carrier energy. Numerically these integrals are easily evaluated as sums over all hopping events $i$ in the time $t$ :

$\int_{0}^{t} \int j(E) d E d t=\frac{1}{A L} \sum_{i} q \Delta z_{i} \quad$ and $\quad \int_{0}^{t} \int E j(E) d E d t=\frac{1}{A L} \sum_{i} E_{i} q \Delta z_{i}$

with $A$ and $L$ the cross section and length of the simulation box and $\Delta z_{i}$ the displacement of the $i$-th hopping event in the direction of the electric field that is applied along $z$. In the above, 


\section{WILEY-VCH}

the energy $E$ of a hop is determined as the average of the initial and final site energies, i.e. $E=\frac{E_{i}+E_{f}}{2}$ such that back and forth hops between neighboring sites and closed loops do not contribute to the sums Eq. 15 and therefore not to the energy transport and Seebeck coefficient.

The Fermi energy $E_{F}$ is calculated by fitting a Fermi-Dirac distribution to the ratio of the DOOS (density of occupied states) and DOS, which are calculated as the time-averaged histograms over all site energies $E_{i}$ (Eq. 12) in the simulation box and all occupied site energies, respectively.

\subsubsection{Model comparison}

In Figure 2 the analytical and kinetic Monte Carlo hopping models introduced above are compared for a representative set of input parameters. Due to excessive computation times, the data points for VRH of doping induced charges at higher doping concentrations are missing. Overall, the analytical model quasi-quantitatively reproduces the numerically exact kMC simulations, showing similar trends with some deviations in numerical values. For virtually all data points, the thermopower as calculated by the approximate expression Eq. 10 (thick solid lines) overestimates the corresponding $\mathrm{kMC}$ result (solid symbols). We attribute this to the fraction of hops actually being to lower final site energies than the characteristic energy $E^{*}$.

For the calculations where charges stem from dopants, i.e. where DOS broadening must be accounted for, kMC simulations have been run with both full updates of site energies (solid lines, filled symbols) and with the first reaction approximation (dashed lines, open symbols). For mobility, the first reaction method seems to give a rather accurate approximation up till relative doping levels of $\sim 10^{-2}$; for thermopower, deviations become significant at much lower concentrations, which we attribute to the DOS and DOOS becoming inaccurate when not all site energies are properly updated, causing additional deviations in $E_{F}$. 


\section{WILEY-VCH}
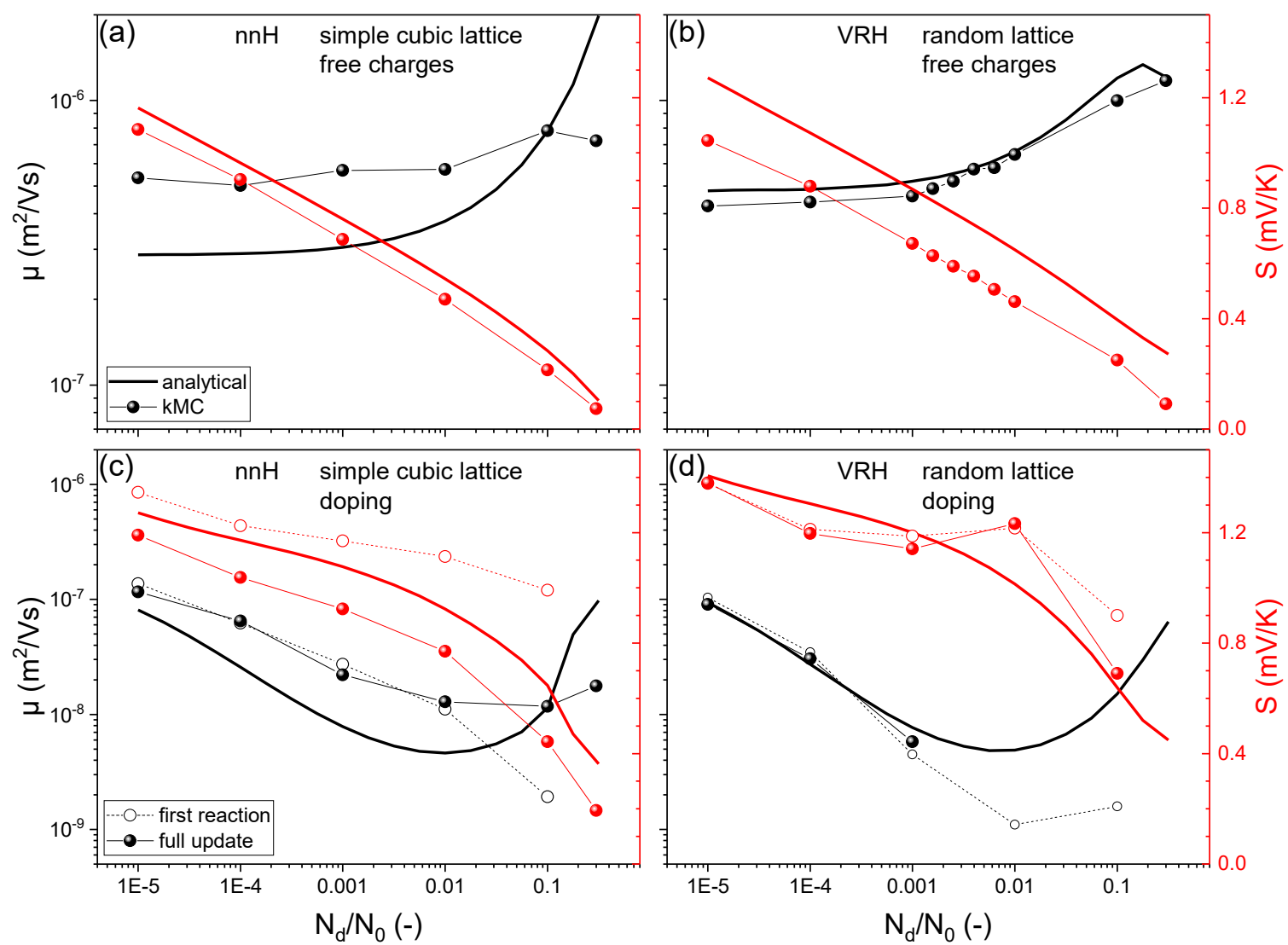

Figure 2. Comparison of mobility (black, left y-axes) and thermopower (red, right y-axes) vs. doping concentration calculated from the analytical hopping models (thick solid lines, Eqs. 3$10)$ and $\mathrm{kMC}$ models (thin lines with symbols) discussed in the text. Panels $(\mathrm{a}, \mathrm{c})$ and $(\mathrm{b}, \mathrm{d})$ are for nearest neighbor hopping and variable range hopping, respectively. Filled and open symbols indicate full updates and first reaction in kMC. Parameters used are $\sigma_{D O S}=60 \mathrm{meV}$, $T=300 \mathrm{~K}, a_{N N}=1.8 \mathrm{~nm}, \varepsilon_{r}=3.6, \alpha \times a_{N N}=0.2$ and $\Delta E=0$.

\section{Material blend design for organic thermoelectrics}

\subsection{DOS engineering in organic semiconductor blends}




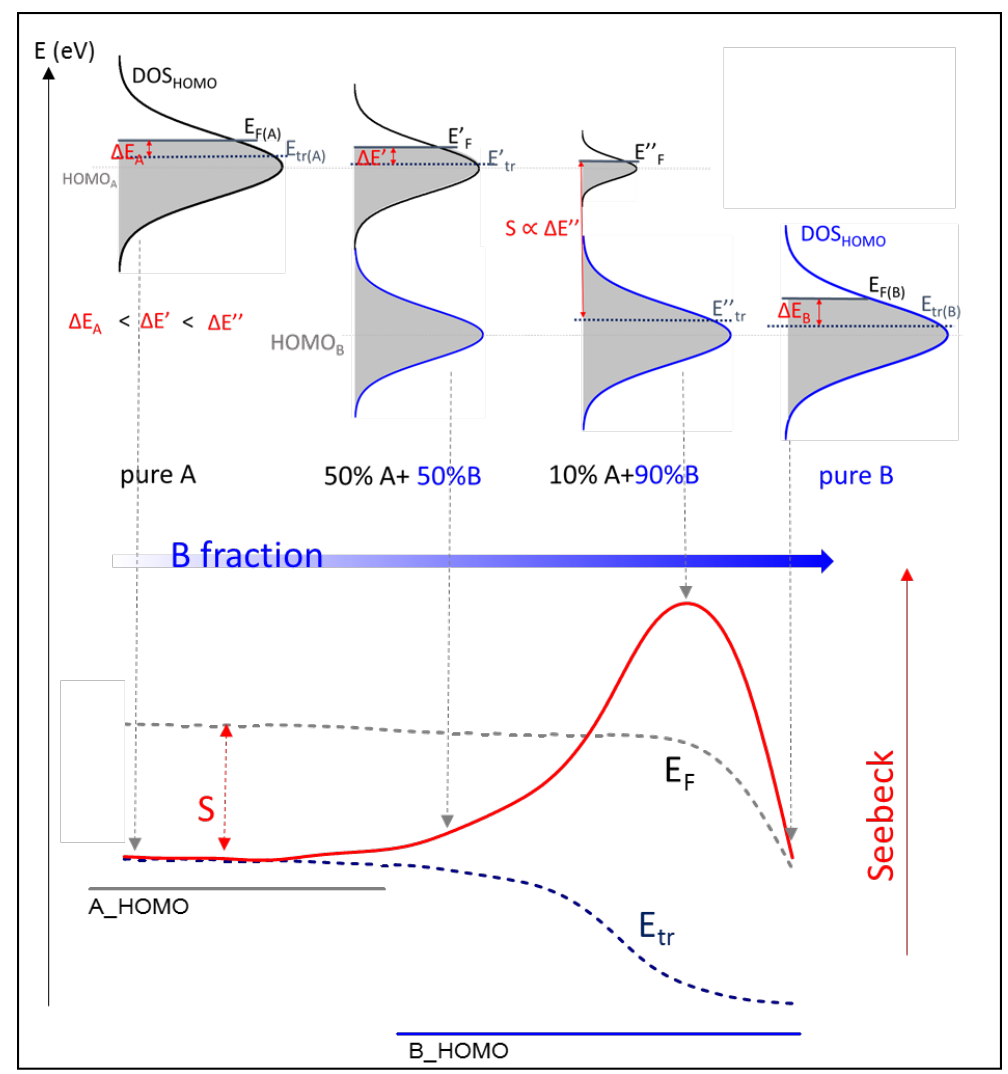

Figure 3. Illustration of DOS engineering to achieve a high Seebeck coefficient $S$. Upper part: hole DOS for a binary mixture A:B where materials A and B have different HOMO energies. White and shaded regions indicate hole- and electron-filled parts of the DOS, respectively. Lower part: corresponding relative positions of the Fermi energy $E_{F}$ and the transport energy $E_{t r}$ and the Seebeck coefficient $S$ that is proportional to their difference. Reproduced with permission. ${ }^{[56]}$ Copyright 2017, Wiley-VCH.

As explained above, thermoelectric properties like the Seebeck coefficient $S$ and the electrical conductivity $\sigma$ can be characterized by a typical or characteristic hop taking place between the Fermi energy $E_{F}$ and transport energy $E_{t r} \cdot{ }^{[41,57]}$ At a given temperature $T$, these relationships can simply be expressed as $S \propto\left(E_{F}-E_{t r}\right) / T$, and $\sigma \propto \exp \left(\frac{E_{F}-E_{t r}}{T}\right)$, where $E_{F}$ is mainly determined by the charge carrier concentration and shape of the DOS and the transport energy $E_{t r}$ is related to the part of the total DOS that facilitates the bulk of the charge transport. ${ }^{[56]}$ On basis of the model introduced in section 2.1, the net DOS distribution is affected by the ionized dopants in the conjugated material. Particularly at high doping concentrations, charge transport takes place entirely in the dopant ion-induced tail of the DOS, see Figure 1a. In other words, the DOS broadening results in a negligible effect of the pristine DOS of the 


\section{WILEY-VCH}

material. ${ }^{[48]}$ Consequently, the thermoelectric properties become independent of pristine disorder, which severely limits the possibilities to optimize the thermoelectric properties of single, doped OSCs beyond obtaining an as high as possible doping level without deteriorating the morphology. ${ }^{[13,58]}$

Given the limited ability to tune the thermoelectric properties of a system containing a single semiconductor, the question of how blends might be used to optimize thermoelectric properties arises naturally. In a seminal paper, Mahan and Sofo theoretically proposed the concept of a delta-shaped transport distribution, situated somewhere above the Fermi energy, to maximize the thermoelectric properties.${ }^{[59]}$ Under the simplifying assumption that no interaction occurs between the intrinsic DOS of the individual OSCs, a similar situation can be engineered in blends of conjugated semiconductors. Zuo et al. revisited the concept to engineer the thermoelectric properties of OSC blends as shown in Figure 3 (referring to hole transport). ${ }^{[56]}$ When a material $\mathrm{B}$ with lower-lying HOMO is added to a material A, a binary mixture A:B is created, exhibiting a double-peaked DOS. At small to intermediate fractions of material $\mathrm{B}$, the holes remain entirely on material $\mathrm{A}$, and therefore both $E_{F}$ an $E_{t r}$ are still located on the A-part and only a small increase in $S$ is observed resulting from the decreased number density in the DOS of material A that supports the charge and energy transport. ${ }^{[56]}$ Further increasing the fraction of material $\mathrm{B}$ in the blend leads to a relative decrease of the number of A-sites, which at some point can no longer contain the optimal percolating network, resulting in $E_{t r}$ gradually shifting into the B-part of the DOS. Simultaneously, $E_{F}$ remains in the A-part of the DOS for any practical A loading, i.e. as long as the concentration of material A is considerably larger than that of the dopant. This is depicted in Figure 3 in the panel labeled ' $10 \% \mathrm{~A}+90 \% \mathrm{~B}$ ', where a maximum in $S$ occurs due to the large gap between $E_{F}$ and $E_{t r}$. Note that the B-part of the DOS is not a delta-function as in the original Mahan and Sofo paper but is broadened due to the disordered nature of OSCs, which will somewhat compromise the maximum attainable performance. Nevertheless, one can engineer the 


\section{WILEY-VCH}

Seebeck coefficient by tuning the blends of two selected OSCs with different HOMO levels.

Note that the maximum value of the Seebeck coefficient depends on the HOMO energy gap

of the two blend materials, with larger energy differences resulting in higher maximum values of $S$.

\subsection{1. p-type blends}

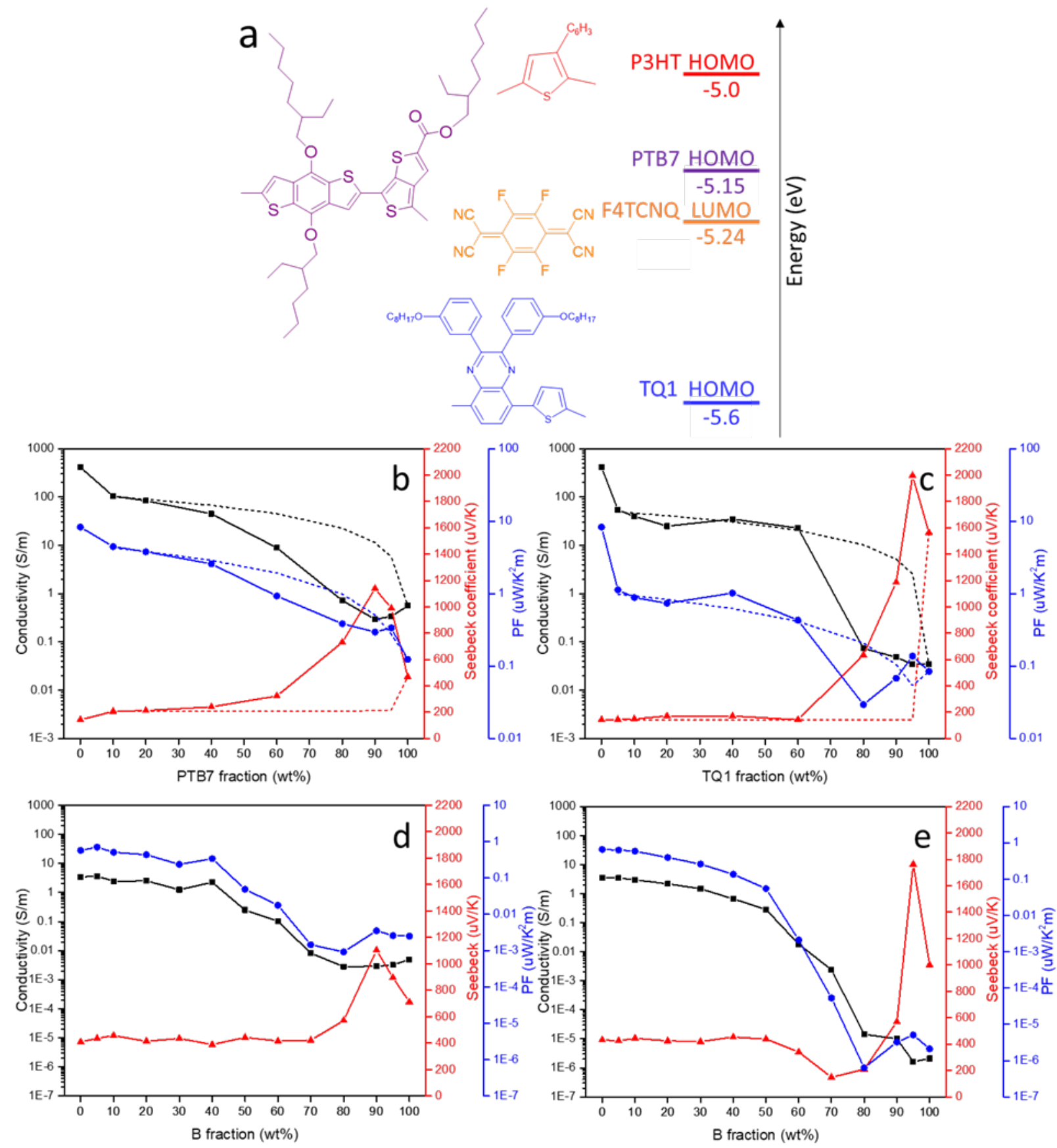

Figure 4. (a) Chemical structure and relevant energy levels of the p-type materials used in investigation of high thermopower in two-component blends. Experimental conductivity, Seebeck coefficient and power factor for (b) P3HT:PTB7 and (c) P3HT:TQ1. Corresponding $\mathrm{kMC}$ simulated results for (d) $\Delta E_{\text {Номо }}=0.3 \mathrm{eV}$ and (e) $\Delta E_{\text {Номо }}=0.5 \mathrm{eV}$ with parameters: 


\section{WILEY-VCH}

doping concentration $c=10^{-2}$; the attempt to hop frequency $v_{0}=10^{-13} \mathrm{~s}^{-1}$; intersite distance $a_{N N}=1.8 \mathrm{~nm}$; Gaussian disorder $\sigma_{D O S}=0.075 \mathrm{eV}$; temperature $T=300 \mathrm{~K}$;

$\mathrm{HOMO}_{\mathrm{A}}-\mathrm{LUMO}_{\text {dopant }}=0.24 \mathrm{eV}$. Reproduced with permission. ${ }^{[56]}$ Copyright 2017, Wiley$\mathrm{VCH}$.

Inspired by Mahan and Sofo, ${ }^{[59]}$ Sun et al. blended a small fraction of P3HTT with F4TCNQ bulk-doped P3HT, where the film was formed from a common solution mixing the OSCs and dopant together. Increased Seebeck coefficients between 580 and $700 \mu \mathrm{V} / \mathrm{K}$ were obtained, while the electrical conductivity of pristine $\mathrm{P} 3 \mathrm{HT}$ was preserved, ${ }^{[60]}$ experimentally confirming the concept of DOS engineering. However, the achieved PFs were still is too low for practical applications due to the low electrical conductivity.

Later research has demonstrated that sequential doping can yield higher electrical conductivities than the conventional doping method using a common solution by preserving film morphology. ${ }^{[13,17,58]}$ Sequential doping is done by spin-coating a dopant-containing solution over the pristine active layer. ${ }^{[61,62]}$ Revisiting the concept of DOS engineering, Zuo et al. used the archetypical P3HT as material A (Figure 4) and mixed it with selected conjugated polymers PTB7 and TQ1 (materials B), having different HOMO energy levels. ${ }^{[56]}$ Sequential (p-type) doping by F4TCNQ was used to improve the electrical conductivity of the blend, leaving the morphology unchanged - the role of the morphology will be discussed in section 3.2 below. The HOMO energy levels of the used materials and the thermoelectrical properties of mixtures of P3HT:PTB7 and P3HT:TQ1 are shown in Figure 4(a).

The HOMO level of P3HT $(-5.0 \mathrm{eV})$ is situated above the LUMO level of F4TCNQ (-5.24 eV). ${ }^{[63]}$ This results in an efficient electron transfer from the HOMO of P3HT to the LUMO of F4TCNQ and gives neat, unblended P3HT the highest electrical conductivity of over $400 \mathrm{~S} / \mathrm{m}$ and a correspondingly low Seebeck coefficient of around $142 \mu \mathrm{V} / \mathrm{K} .{ }^{[56]}$ Since the HOMO levels of PTB7 $(-5.15 \mathrm{eV})$ and TQ1 $(-5.6 \mathrm{eV})$ sit around or below the LUMO of F4TCNQ, the corresponding electrical conductivity of the pure materials decreases, whereas the Seebeck coefficient increases. As anticipated, Figure 4(b,c) shows a peak in $S$ for both material systems, 


\section{WILEY-VCH}

occurring at an A:B ratio of around 10:90. The higher peak value for $S$ found for $\mathrm{P}_{3} \mathrm{HT}_{0.05}: \mathrm{TQ}_{0.95}, S \approx 2000 \mu \mathrm{V} / \mathrm{K}$, than for $\mathrm{P}_{3} \mathrm{HT}_{0.1}: \mathrm{PTB}_{0.9}, S \approx 1100 \mu \mathrm{V} / \mathrm{K}$, is due to the deeper lying HOMO of TQ1 than that of PTB7. ${ }^{[56}$ Likewise, in P3HT:TQ1 blends, the maximum $S$ is found at a smaller A-fraction (5\% of P3HT in weight) than in P3HT:PTB7 blends, which is due to the larger energy penalty for hopping to material B, i.e. charge carriers 'try to stay longer' in material A, keeping the transport level closer to the Fermi energy.

The dashed lines in Figure 4(b,c) show the interpolation between the binary extremes using an effective medium model in which the system is assumed to consist of two non-interacting subphases. The apparent absence of a peak in this model indicates that the peaking Seebeck coefficient is not a straightforward blending effect, but results from the electronic interaction between the two conjugated polymers. In contrast, a near-quantitative description of the experiments could be given by a kinetic Monte Carlo model based on nearest neighbor hopping in random A:B blends of varying composition, see panels (d) and (e) of Figure 4, and section 2.2 above. ${ }^{[56]}$

\subsection{2. n-type blends}
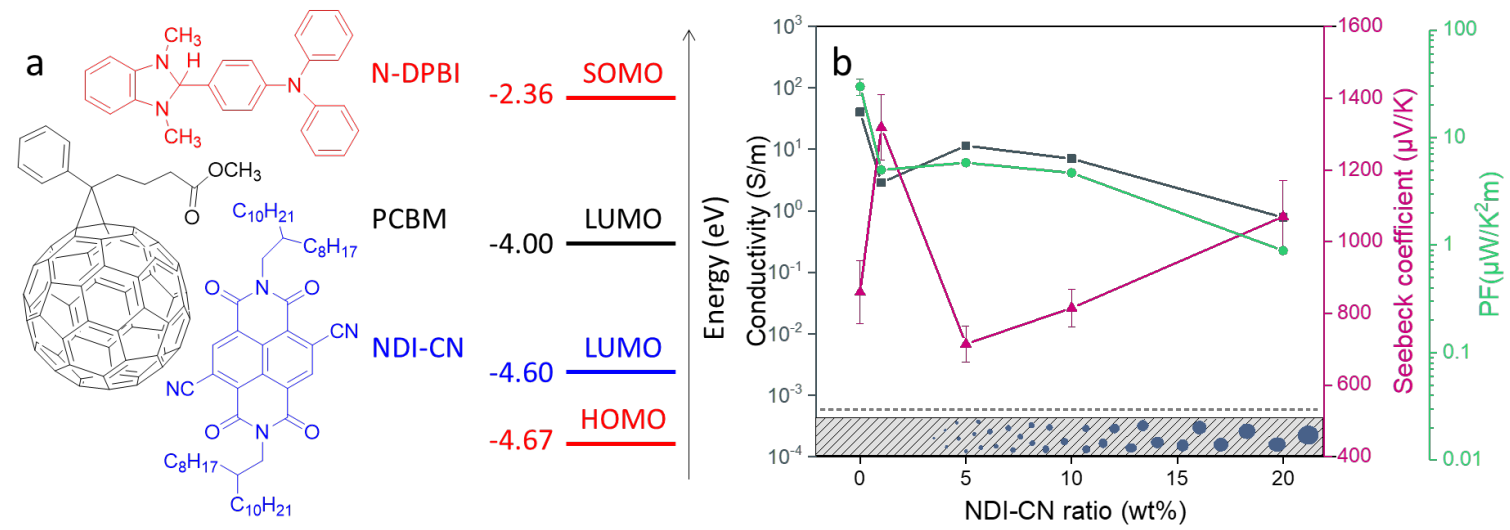

Figure 5. (a) Chemical structure and relevant energy levels of the n-type materials used in investigation of high thermopower in two-component blends. (b) experimental conductivity, Seebeck coefficient and power factor (PF) vs. NDI-CN guest concentration. The dashed line represents the electrical conductivity of doped neat PCBM. Bottom inset: schematic morphology for the blending system. Reproduced with permission. ${ }^{[64]}$ Copyright 2018, Wiley$\mathrm{VCH}$. 


\section{WILEY-VCH}

To exploit the full potential of the available thermal energy, a TEG needs both p-type and ntype materials. ${ }^{[65]}$ In this context it is relevant that the DOS engineering concept demonstrated above is truly universal, i.e. can be used to maximize the thermopower of both p-type and ntype OSCs. A material system for Seebeck coefficient enhancement by blending of n-type OSC has been investigated in Ref. ${ }^{[64]}$ as shown in Figure 5. The deeper LUMO $(-4.67 \mathrm{eV})$ of the small molecule NDI-CN makes it an electron trap when blended with the common n-type material PCBM (LUMO -4.0 eV). Active layers are inverse-sequentially doped by N-DPBI, where the NDCI-CN:PCBM film is spin-coated on top of the dopant layer. A clear peak in thermopower occurs at a fraction of about $1 \%$ of NDI-CN, with a corresponding $S \approx 1400 \mu \mathrm{V} / \mathrm{K}$ and a PF close to $5 \mu \mathrm{Wm}^{-1} \mathrm{~K}^{-2} \cdot{ }^{[64]}$ In line with the trend for $\mathrm{p}$-type materials discussed above, the large LUMO energy difference of $\sim 0.67 \mathrm{eV}$ between material A and B rationalizes that the maximum value of $S$ is observed at low, $\sim 1 \mathrm{wt} \%$, fractions of NDI-CN.

On basis of the described theoretical and experimental work for both p-type and n-type OTE, DOS engineering emerges as an effective way to tune the thermoelectric properties, especially to maximize the thermopower. ${ }^{[56,59,60,64]}$ Unfortunately, at the regions in parameters space where $S$ reaches more extreme values, this comes at the cost of strongly (roughly exponentially) declining electrical conductivities, which leads to considerable decreases in power factor. ${ }^{[56,64]}$ Using kinetic Monte Carlo simulations, as in panels d and e of Figure 4, it was shown in Ref. [56] that for mixed systems $A: B$ in principle the maximum PF always occurs at the pure compound that is most easily doped (A). However, for the p-type blends in Figure 4, at the composition of the peak in $S$, the PF is higher than for pure material B (PTB7 or TQ1), i.e. the presence of the trap A (P3HT) increases the PF of the pure compound B. This suggests that for example the PF of P3HT may be enhanced by blending with a material with a shallower HOMO. Despite the still low power factor, blended materials with a high electronic Seebeck coefficient and still reasonable electrical conductivity might be suitable for low-cost, low-power devices, 


\section{WILEY-VCH}

where absolute voltage and cost are more important than power, e.g. in thermometry or lowpower autonomous sensors. ${ }^{[56]}$

\subsection{Role of morphology}
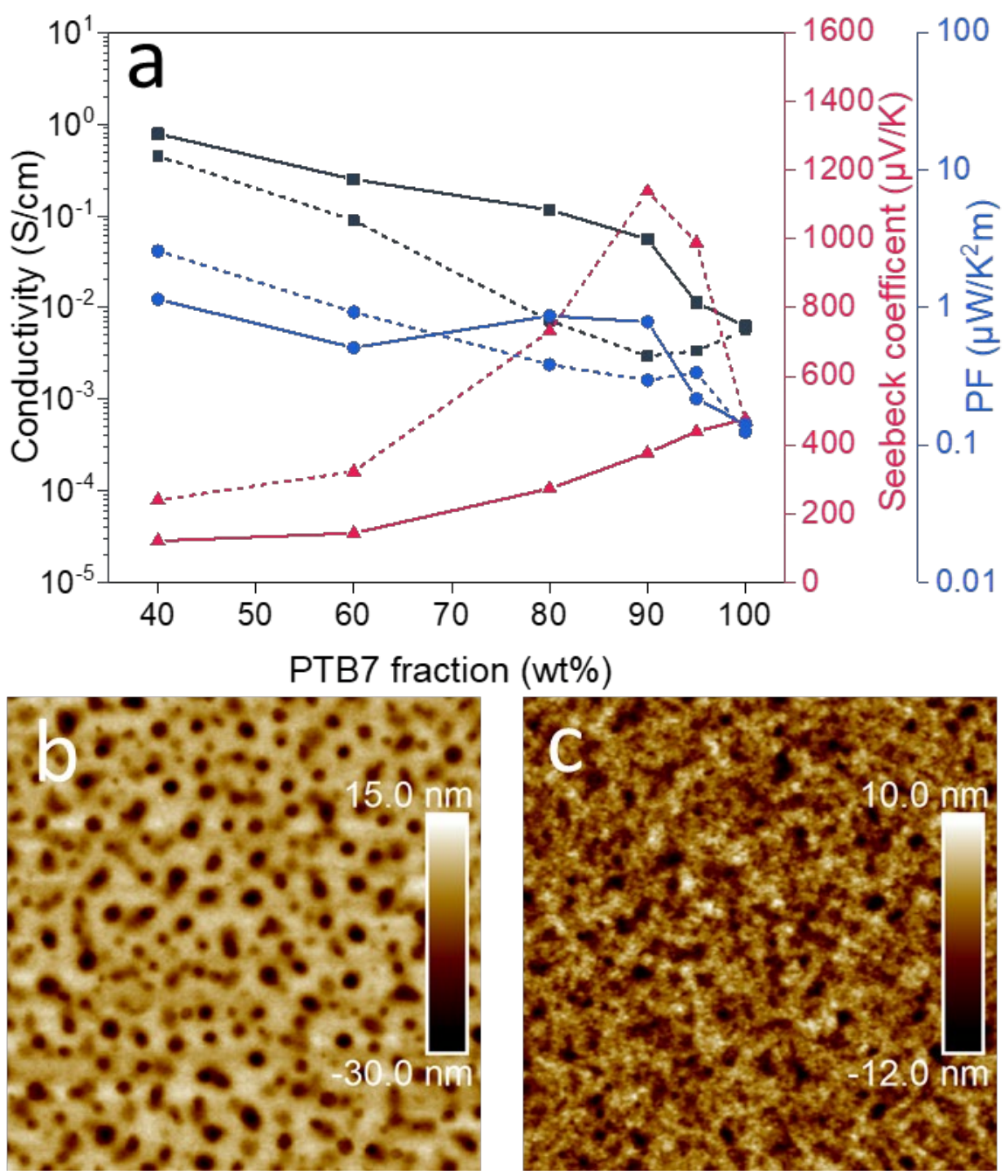

Figure 6. (a) Conductivity, Seebeck coefficient and power factor for P3HT:PTB7 with 5\% DIO as a function of active layer composition. Dashed lines are data from Figure 4(b), i.e. without DIO; AFM image for (b) $\mathrm{P}_{3} \mathrm{HT}_{0.1}: \mathrm{PTB} 7_{0.9}$ without DIO and (c) with 5\%. All sizes $5 \times 5 \mu \mathrm{m}$. Reproduced with permission. ${ }^{[66]}$ Copyright 2018, American Chemical Society.

It is well-established that morphology has a strong influence on the performance of organic electronic devices such as organic solar cells, organic light-emitting diodes, field-effect transistors and so on. ${ }^{[67-71]}$ Especially in organic solar cells, intensive research established a stark correlation between performance and blend morphologies that can be varied during 


\section{WILEY-VCH}

processing by additives, choice of solvent etc. ${ }^{[67,68,72]}$ However, it is still difficult to quantitatively understand the influence of morphology on performance in OSC-based devices. Recent reports indicated that morphology also has a strong influence on the thermoelectric properties of single OTE materials. ${ }^{[13,17,61,62,73,74]}$ For instance, Patel et al. showed that the longrange order of vapor-doped PBTTT strongly enhanced the power factor, reaching up to $120 \mu \mathrm{Wm}^{-1} \mathrm{~K}^{-2}{ }^{[13]}$ Hamidi-Sakr et al. showed that the morphology impacts anisotropic thermoelectric properties and charge transport in a series of doped single polythiophene derivatives with different alkyl side chains. ${ }^{[74]}$

Considering the potentially complicated distribution of localized sites in two-OSCs blends, it is (even) more difficult to understand and predict the relation between morphology and performance in a mixture system. Different blend morphologies, like strongly phase-separated or well-mixed, will cause different behavior, varying with composition and energy difference. In bulk heterojunction solar cells, 1,8-diiodooctane (DIO) is an effective and commonly used additive, which can improve the performance of devices due to a favorable finely mixed morphology formed after adding this additive to the common donor-acceptor solution. ${ }^{[75-80]}$ In Ref. ${ }^{[66]}$ Zuo et al. investigate the effect of the processing agent DIO on the morphology and thermoelectric properties of blended systems. Whereas in section 3.1 a clear peak in thermopower versus composition curve was shown for P3HT:PTB7 blends, the peak disappeared upon adding 5 vol\% DIO to the thermoelectrically optimized blend, as shown in Figure 6(a), leading to a drop of the Seebeck coefficient in $\mathrm{P}_{3} \mathrm{HT}_{0.1}: \mathrm{PTB} 7_{0.9}$ from over $1100 \mu \mathrm{V} / \mathrm{K}$ to $400 \mu \mathrm{V} / \mathrm{K}$ with the DIO additive. Zuo et al. attributed the difference to the film morphology that changed from strongly phase separated to well-mixed, as shown in Figure 6(b,c). Unsurprisingly, this indicates that the blend morphology heavily impacts the thermopower in OSC blends. 


\section{WILEY-VCH}

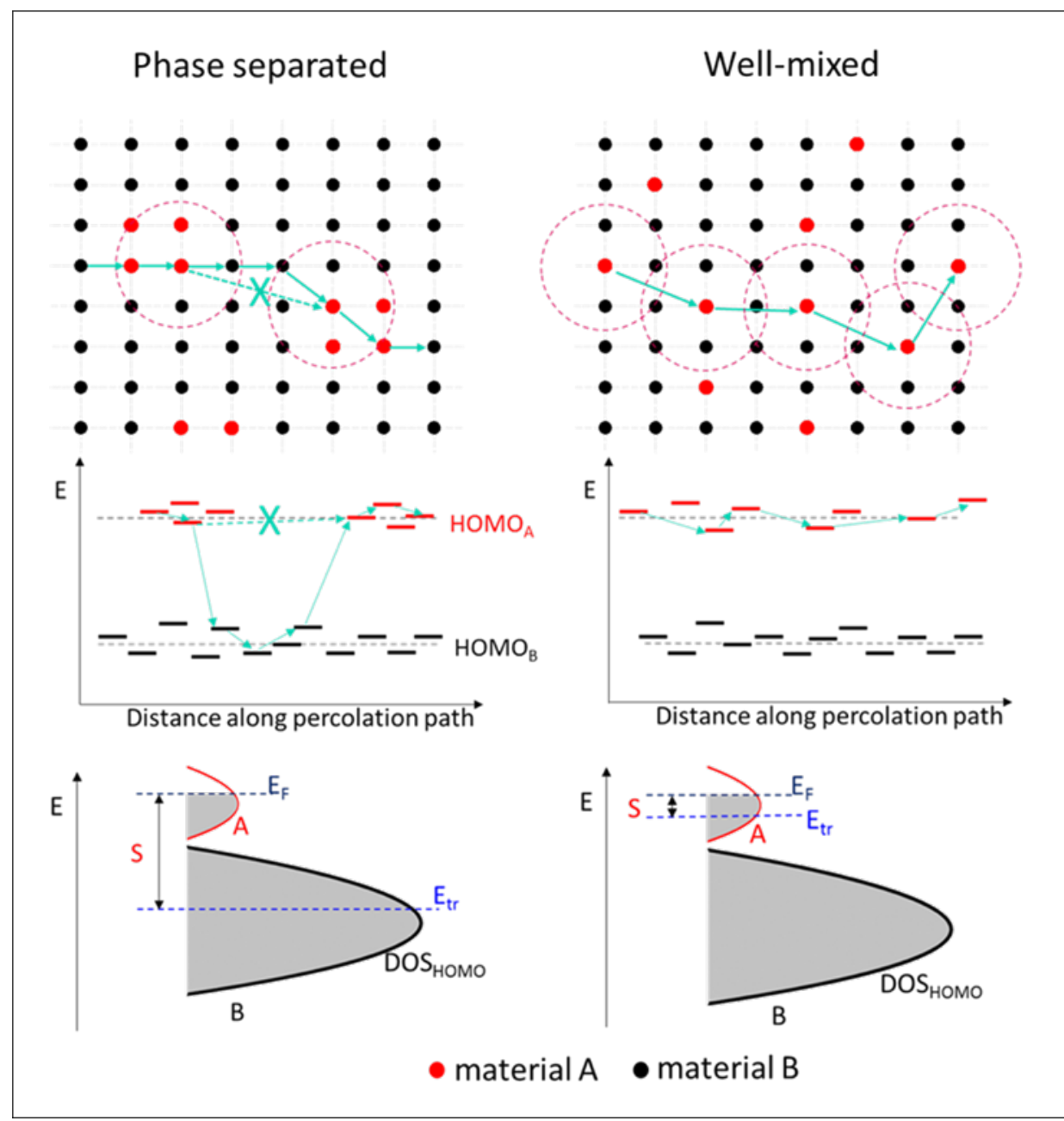

Figure 7. (top) Schematic overview of the site distribution in a 2-component blend (dashed circles indicate the typical hopping distance; green arrows indicate the hopping path); (middle) corresponding energy diagram; (bottom) density of states and characteristic energies, in (left) phase separated and (right) well-mixed morphologies with the same blend ratio. Materials A and B are indicated by red and black colors, respectively. Reproduced with permission. ${ }^{[66]}$ Copyright 2018, American Chemical Society.

As explained above, charge transport via localized sites, which is assumed to occur by thermally activated tunneling (hopping), is the baseline to understand charge and energy transport in OSC. ${ }^{[81]}$ To explain the relation of morphology with thermopower in OSC blends, the mechanism shown in Figure 7 was proposed. ${ }^{[66]}$ In contrast to the nearest-neighbor hopping framework originally used to explain the composition dependence of thermoelectric blends, c.f. Figure 3, explaining the morphology dependence required assuming that charge transport occurs by variable range hopping (VRH). 


\section{WILEY-VCH}

The blend morphologies, as measured by AFM in Figure $6(b, c)$, can be mapped on the schematic morphologies in the top panels of Figure 7. For phase separated morphologies, the clusters formed by material A are distributed far away from each other in material $\mathrm{B}$. Consequently, the percolation pathway must include sites from material B, causing the transport energy to shift towards the DOS of material B. For any practical doping level and A loading, $E_{F}$ will remain in the A-part of the DOS. Hence, the resulting energetics are like the situation described in the previous section, resulting in a high thermopower. In contrast, for the wellmixed morphologies as seen in Figure 7 (right), the sites of material A are, in lowest order, randomly distributed in material $\mathrm{B}$. Then, provided hopping is not constrained to nearest neighbors, i.e. typical tunneling distances can be larger than the average inter-site distance in material A, it is possible for percolation to occur just in material A. In this scenario, both transport energy and Fermi energy are located in the DOS of material A, decreasing both thermopower and power factor. ${ }^{[66]}$
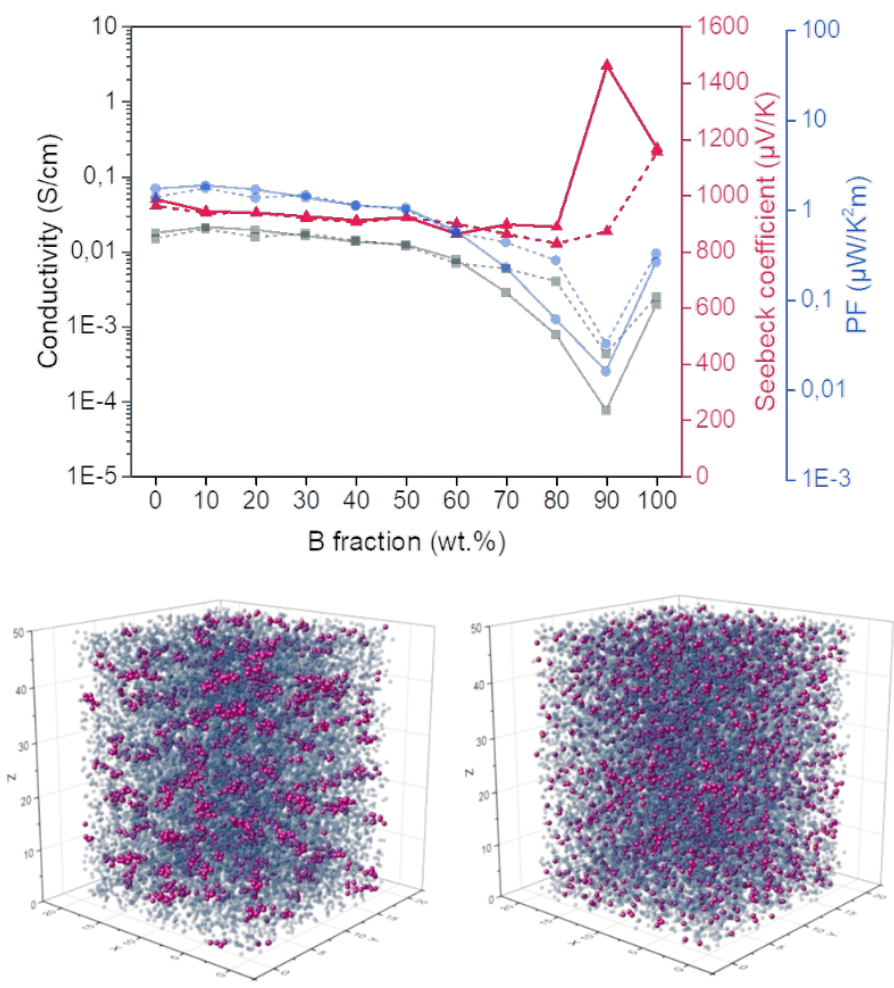

Figure 8. (a) Electrical conductivity, Seebeck coefficient and power factor vs. composition from VRH kinetic Monto Carlo simulations for $\mathrm{A}_{x}: \mathrm{B}_{1-x}$ mixtures with clusters of a single 


\section{WILEY-VCH}

material (solid lines) and without clusters (dashed lines). Panels (b) and (c) show typical morphologies used in the kinetic Monto Carlo simulations for $\mathrm{A}_{10}: \mathrm{B}_{90}$ mixtures $\mathrm{w} /$ and w/o clustering of the A component, respectively. Parameters used are the HOMO energy difference $\Delta E_{\text {HOMO }}=0.3 \mathrm{eV}$ and doping concentration $c=10^{-3}, v_{0}=10^{-13} \mathrm{~s}^{-1}, a_{N N}=1.8 \mathrm{~nm}$, $\sigma_{D O S}=75 \mathrm{meV}, T=300 \mathrm{~K}, \mathrm{HOMO}_{\mathrm{A}}-\mathrm{LUMO}_{\text {dopant }}=0.24 \mathrm{eV}, \alpha=2 \mathrm{e} 9 \mathrm{~nm}^{-1}$; correlation radius of the A compound clusters in units of $a_{N N}$ are $r_{C}=1.5$ or 0 . Reproduced with permission. ${ }^{[66]}$ Copyright 2018, American Chemical Society.

The concept outlined above has been implemented in a kinetic Monte Carlo algorithm for variable range hopping on a random lattice, see Figure 8 and section 2.2 above. Clustered morphologies were generated by the numerical annealing procedure developed by Peumans et al. ${ }^{[82]}$ and later used by Marsh et al. ${ }^{[83]}$ More details of this model can be found in Ref. ${ }^{[66]}$ The calculated thermoelectric properties and corresponding blend morphologies are shown in Figure 8. Despite the fact that the length scale of phase separation in the model was different from that in actual devices where the clusters are around several hundreds of nanometer across, the model was argued to be relevant to the actual system because the percolation problem is largely invariant to absolute length scales. ${ }^{[66]}$ The trends from kMC simulations in conductivity and Seebeck coefficient agree well with those observed in experiments, both for phaseseparated (P3HT:PTB7 w/o DIO) and well-mixed (P3HT:PTB7 w/ DIO) morphologies. Particularly, the reproduced and distinct behavior of the presence or absence of a peak in $S$ for different morphologies gives credence to the mechanism proposed in Figure 7.

\subsection{Blends with insulating polymers}

Chemical or electrochemical doping of polymeric semiconductors, as discussed in the previous sections, is the most common technique to increase electrical conductivity by increasing charge carrier concentration. As discussed above, these methods are typically subject to the inverse relationship between electrical conductivity and Seebeck coefficient. However, it has been shown, already more than 20 years ago, that blending of certain conjugated polymers with appropriate insulating polymers, also referred to as dilution, can lead to an improvement of the 


\section{WILEY-VCH}

electrical conductivity on the same order as conventional chemical doping, ${ }^{[48,84-86]}$ while leaving the Seebeck coefficient relatively constant. This results in an improved PF and thermoelectric figure of merit ZT over doped systems. ${ }^{[85,87-91]}$ Additionally, blending of conductive polymers with high-melting point insulators allows for the engineering of processability and mechanical and optical properties of the blends, opening up new applications for OTEs. ${ }^{[89,90]}$ While there exists a reasonably large amount of literature studying the morphology and opto-electrical properties of diluted polymer blends, investigations of the thermoelectric performance of such OSC/insulator blends are rather rare, but, nonetheless, show promise for future applications and further research. In this section a brief overview of the available literature is given.

Early studies include temperature dependent conductivity and thermopower investigations of polyaniline-camphor-sulfonic acid (PAni-CSA) blended with insulators poly(methyl methacrylate) (PMMA), ${ }^{[84,85,92]}$ polyaniline in its emeraldine salt form, protonated with an organic acid (PAni-ES) blended with PMMA or glycol-modified polyethylene terephthalate (PTEG), ${ }^{[87]}$ as well as bis(2-ethyl hexyl)hydrogen phosphate doped (DiOHP-doped) PAni blended with polystyrene (PS). ${ }^{[86]}$ Table 1 summarizes thermoelectric parameters of these and related works discussed below. These studies show that both conductivity and thermopower increase upon increasing the volume fraction of the insulator with a maximum around $60 \mathrm{wt} \%$ insulator. The increase in conductivity is explained by the formation of a self-assembled, interpenetrated percolation network of crystalline PAni nanowires (NWs) embedded in the insulating polymer matrix. This network exhibits a low percolation threshold (0.6-1 wt \%), and a combination of metallic transport in the PAni NWs and hopping transport between the NWs (i.e. tunneling through the insulator). Interestingly, the power factor of CSA-doped PAni/PMMA blends and PAni-ES/insulator blends show surprisingly large power factors of up to $1.7 \mu \mathrm{Wm}^{-1} \mathrm{~K}^{-2},{ }^{[84,87]}$ a fact which has found little to no attention in subsequent literature. 


\section{WILEY-VCH}

However, thermoelectric properties of pure PAni/insulator blends are of purely academic nature.

In a more recent study, Lu et al. investigated blends of oxygen-doped poly(3-butylthiophene) (P3BT) and PS and found that if P3BT is allowed to crystallize into NWs before vitrification with PS, an increase of conductivity is observed, peaking between $40-60 \mathrm{wt} \%$ of PS. ${ }^{[89]}$ The main reason for the conductivity increase is found in the reduction of the activation energy for hopping transport upon forming a large interface between the P3BT NWs and the insulating matrix. ${ }^{[93]}$ Interestingly, the Seebeck coefficient also shows an increase with PS content, albeit a minor one, and peaks around the same wt $\%$-range. This results in a PF of $7.2 \mu \mathrm{Wm}^{-1} \mathrm{~K}^{-2}$, which is an order of magnitude higher than for unblended P3BT. The improvement of the thermoelectric properties of this blend is again attributed to the quasi-1D charge transport within the interpenetrated NW percolation network that is homogeneously dispersed in the PS matrix. It is argued that despite the decrease of activation energy with PS content, the Seebeck coefficient remains relatively constant due to the balancing effect of an increased local thermoelectric potential in quasi-1D charge transport. However, no further insight into the physics behind this phenomenon is presented.

Lu et al. also investigated a ternary system, where P3BT is doped by 2,3,5,6-tetra- fluoro7,7,8,8-tetracyanoquinodimethane (F4TCNQ), rather than by oxygen, before being blended with PS. ${ }^{[89]}$ They find that the affinity for agglomeration by F4TCNQ-doping inhibits the formation of an interpenetrated NW network and that, while the conductivity increases with PS and, of course, F4TCNQ content, the Seebeck coefficient decreases leading to inferior thermoelectric performance.

Additionally, the boundaries between the P3BT grains and the PS matrix are argued to deteriorate phonon transport, which leads to a smaller thermal conductivity and consequently a larger ZT. In fact, the phonon scattering at the boundaries between conductive filaments in an insulating matrix appears to be the main engineering guideline for nano-composites 


\section{WILEY-VCH}

including carbon nano-tubes, -sheets and -wires embedded in a matrix of insulating

polymers. ${ }^{\left[{ }^{94-97]}\right.}$ In view of the absence of long-range lattice vibrations (phonons) in structurally disordered polymers and blends thereof, it is uncertain to which degree interfacial effects are relevant to suppressing the thermal conductivity of these systems.

Wang et al. theoretically studied the thermoelectric properties of quasi-1D charge transport in molecular nanowires based on an evaluation of the Kubo formalism using the Holstein model.${ }^{[88]}$ In a theoretical study, they apply their model to P3HT and PEDOT NWs and find that inter-site coupling has the strongest effect on thermoelectric properties of molecular NWs, where stronger coupling leads to higher power factors. This finding can qualitatively be compared to the results discussed above for bulk materials (sections 1 and 2) that increasing conductivity at the expense of a lower thermopower typically leads to higher PF in organic thermoelectrics.

Kiefer et al. investigated the thermoelectric properties of poly(3-hexylthiophene) (P3HT) doped with F4TCNQ and embedded in an insulating matrix of semi-crystalline poly(ethylene oxide) (PEO). ${ }^{[90]}$ Doping of the P3HT:PEO blend with F4TCNQ occurred in solution, which was then drop-casted at an elevated temperature $\left(110^{\circ} \mathrm{C}\right)$ to form freestanding films with thicknesses of around $30 \mu \mathrm{m}$ and homogeneous morphology. Conductive AFM investigation concludes that the lateral distribution of the conductive phase is continuous and consists of highly conductive domains that are surrounded by a less conductive matrix. The thermoelectrical properties are studied as a function of both F4TCNQ concentration in P3HT and PEO content in the ternary P3HT:F4TCNQ:PEO blend. The authors find that the power factor of $10^{-1} \mu \mathrm{Wm}^{-1} \mathrm{~K}^{-2}$ of the "pure" P3HT:F4TCNQ blend, that is observed irrespective of F4TCNQ load, can be maintained up to a PEO content of around 60wt $\%$ for an F4TCNQ concentration of $20 \mathrm{wt} \%$. The power factor for an F4TCNQ load of 5wt $\%$ decreases upon adding PEO to the blend. The authors attribute this behavior to the combination of the large Seebeck coefficient from the less conductive matrix and the high conductivity from the 


\section{WILEY-VCH}

percolation network formed by the more conductive P3HT:F4TCNQ domains. Together with a relatively invariant thermal conductivity $\kappa=0.3 \mathrm{Wm}^{-1} \mathrm{~K}^{-1}$, the authors estimate a $\mathrm{ZT}=10^{-4}$ for their ternary blends. While this value is not higher than what can be estimated from other literature on pure P3HT:F4TCNQ films, these ternary films exhibit superior mechanical properties and much larger thicknesses. Both aspects are key to actual device applications; especially thicknesses beyond the micrometer range are a prerequisite to maintain any appreciable temperature difference across a TEG.

The authors compare their results to the empirical slope-1/4 powerlaw relationship between thermopower and conductivity found for many doped organic semiconductors ${ }^{[11,48,98]}$ and find that only samples with more than $15 \mathrm{wt} \%$ P3HT:F4TCNQ come close to the trend predicted for not-mobility limited systems. ${ }^{[11]}$ However, upon inspection of the data (SI in Ref. ${ }^{[90]}$ ) it can be seen that none of the data shows a power-law trend. This indicates that the relation between charge and energy transport in OSC/insulator or, by extension, doped OSC/insulator blends, as a function of insulator loading, is governed by fundamentally different mechanisms than in pure OSC as a function of (chemical) doping, which is not surprising. Plotting the data in Table 1 in the same way as Figure 1 b produces a very scattered graph (not shown) that does not allow to draw any meaningful conclusions.

Table 1: Selected thermopower, electrical conductivity and power factor values for various OSC/insulator blends extracted from literature discussed in the text. Values are either taken from tables found in the reference materials or best estimates from graphs in the reference material).

$\begin{array}{llllll}\text { OSC/Matrix } & \begin{array}{l}\text { OSC } \\ (\mathrm{wt} \%)\end{array} & \begin{array}{l}\text { Thermopower } \\ (\mu \mathrm{V} / \mathrm{K})\end{array} & \begin{array}{l}\text { Conductivity } \\ (\mathrm{S} / \mathrm{cm})\end{array} & \begin{array}{l}\text { Power } \\ \text { Factor } \\ \left(\mu \mathrm{Wm}^{-1} \mathrm{~K}^{-}\right.\end{array} & \text {Ref. } \\ & & & & 2) & \\ \text { PAni:CSA } & 100 & \sim 8 & 226 & \sim 1.45 & {[84]} \\ \text { PAni:CSA/PMMA } & 67 & \sim 8.3 & 109 & \sim 0.75 & {[84]} \\ \text { PAni-ES/PMMA } & 40 & \sim 9.5 & 30 & \sim 0.27 & {[87]} \\ \text { PAni-ES/PTEG } & 40 & \sim 8 & 3.6 & \sim 0.023 & {[87]} \\ \text { PAni-ES } & 100 & \sim 3.5 & 18 & \sim 0.022 & {[87]} \\ \text { PAni/PS } & 20 & \sim 8.4 & 4.6 & \sim 0.033 & {[86]} \\ \text { PAni/PS } & 10 & \sim 9 & 2.1 & \sim 0.017 & {[86]} \\ \text { PAni/PS } & 2 & \sim 8.25 & 0.15 & \sim 1 \times 10^{-3} & {[86]}\end{array}$




\section{WILEY-VCH}

$\begin{array}{llllll}\text { P3BT } & 100 & \sim 540 & 2.7 \times 10^{-4} & \sim 7.9 \times 10^{-3} & {[89]} \\ \text { P3BT/PS } & 40 & \sim 610 & 1.8 \times 10^{-4} & \sim 0.067 & {[89]} \\ \text { F4TCNQ(5\%):P3HT/PS } & \sim 40 & \sim 180 & \sim 1.5 \times 10^{-4} & \sim 4.9 \times 10^{-4} & {[90]} \\ \text { F4TCNQ(20\%):P3HT/PS } & \sim 40 & \sim 60 & \sim 0.28 & \sim 0.1 & {[90]}\end{array}$

Tentatively distilling a design rule from the findings above, blending with otherwise inert insulating polymers seems to be a method that, if judiciously implemented, can give rise to a filamentary network of the conjugated phase with improved connectivity and charge transport and a more or less constant thermopower. In other cases, the theoretically expected tradeoff between conductivity and thermopower is obtained. In absence of a substantial body of data, the impact on (the lattice contribution to) the thermal conductivity of this type of blending is somewhat unclear, but the absence of long-range lattice vibrations (phonons) in disordered organic matter make significant effects unlikely.

\subsection{PEDOT:PSS}

Poly(3,4-ethylenedioxythiophene) PEDOT has quickly developed into the most studied material for organic thermoelectric applications owing to its easy processability, ubiquitous availability and, for an organic material, high thermoelectric power factor. Since PSS poly(4styrenesulfonate) is electronically insulating, PEDOT:PSS could, in that respect, be considered a special case of a blend of a conjugated and a non-conjugated polymer as discussed in section 3.3 above. However, PSS also acts as a template for the oxidative polymerization of EDOT into PEDOT and as an (electrostatic) stabilization of the positive charge of the hole polarons on PEDOT. The ionization fraction of PEDOT:PSS is around 30\% and relatively large loadings of insulating PSS are typically needed to stabilize these charges and to achieve processability from water-borne suspensions. The fact that PSS is also ionically conductive furthermore leads to mixed conductivity phenomena at high relative humidity, a topic that will not be further discussed here. ${ }^{[99,100]}$ For these reasons it can upfront 


\section{WILEY-VCH}

be expected that PEDOT:PSS will behave differently than the blends in section 3.3 where the second component is more inert. Table 2 provides an overview of selected results.

It is generally accepted that charge transport in PEDOT:PSS occurs via either quasi-1D VRH through PEDOT-rich filaments, ${ }^{[101,102]}$ or 3D VRH through PEDOT-rich pancake-shaped clusters $^{[103,104]}$ that in all cases are embedded in a matrix consisting mostly of excess PSS with low (ionic) conductivity. The limiting process in charge transport in pristine PEDOT:PSS is therefore the hopping between PEDOT-rich domains as opposed to the hopping within these domains. Due to the lamellar nature of typical PEDOT:PSS morphologies, conductivities tend to be highly anisotropic, which can also be understood in a VRH framework..$^{[103-105]}$ As no reliable data are available regarding anisotropy in thermopower, this topic will not be discussed and we will focus on in-plane geometries. It should, moreover, be kept in mind that there exist large differences in formulations and associated morphologies of PEDOT:PSS and one can therefore not automatically generalize individual results to the whole material system. The conductivity of PEDOT:PSS can be improved greatly by so-called secondary doping methods, mainly involving mixing the aqueous PEDOT:PSS solution with high boiling solvents (HBS). These 'doping' methods typically do not increase charge carrier concentration and thereby circumvent the conventional inverse conductivity-thermopower relationship. Especially polar solvents with a high dielectric constant such as ethylene glycol (EG) or dimethyl sulfoxide (DMSO) are popular candidates for secondary doping of PEDOT:PSS and have consistently been shown to increase the electrical conductivity by several orders of magnitude, ${ }^{[106]}$ without effecting the thermopower.

The effects of HBS-treatments on morphology and the origin behind this steep conductivity increase have been studied in numerous scientific publications over the last $15+$ years and several mechanisms have been suggested. Blending high dielectric constant polar solvents in the aqueous PEDOT:PSS solution screens the positive charge on the PEDOT chains from the negative charge on the PSS counter ion. ${ }^{[106,107]}$ This screening effect is believed to be 


\section{WILEY-VCH}

increased by the addition of a salt in a cosolvent. ${ }^{[108]}$ Using sorbitol as an additive in the solution has been shown to promote separation and aggregation of PEDOT and PSS, also leading to greatly increased conductivities. ${ }^{[109-111]}$ Furthermore, several publications point out that HBS-solution-treatment improves the crystallinity of PEDOT grains and leads to a spontaneous alignment of the PEDOT chains along a preferred (in-plane)

direction. ${ }^{[91,107,110,112]}$ This reduces the inter-chain hopping distances and improves charge transport predominantly in the in-plane direction. However, in their investigation of HBStreated PEDOT:PSS, van de Ruit et al. argue that the HBS-solution-treatment does not significantly change the bulk morphology of the film. ${ }^{[102]}$ Rather, the conductivity increase can be related to either an increase in the interconnectivity between the PEDOT-rich filaments or to a reduction of the spread between conductivities of the filaments. Evidence for this is given by a TEM analysis, in which no morphological change is observed upon HBStreatment, as well as on an analysis of the characteristic temperature and reduced activation energy. The latter shows that transport in HBS-treated PEDOT:PSS films reaches the critical regime preceding a metal-insulator transition and is thus not limited by percolation anymore, as evidenced by the power-law temperature dependence and a small characteristic temperature $T_{0}$ of the VRH formalism, which was also observed in other works. ${ }^{[102,106,113]}$

Another way of improving charge transport, as measured by the conductivity, is by selectively removing PSS from PEDOT:PSS (selective dedoping). This can be done by treatment of the PEDOT:PSS film with hydrophilic HBS like DMSO or EG, which dissolve part of the PSS.$^{[7,107,114,115]}$ Another effective way to remove PSS from PEDOT:PSS is by washing the film in $\mathrm{H}_{2} \mathrm{SO}_{4} \cdot{ }^{[91,102,116,117]}$ There are several effects of PSS removal on PEDOT:PSS described in literature. Removing an amount of insulator increases the relative amount of conducting material, leading to an overall higher conductivity. Additionally, it is believed that PSS removal leads to phase segregation and induces conformational changes in the PEDOT chains from coil-like to a more charge transport-friendly linear (fibrillar) configuration, somewhat 


\section{WILEY-VCH}

similar to the effect of mixing HBS in the PEDOT:PSS solution. ${ }^{[91,102,108,117]}$ In fact, while HBS-solvent-treatment only effects the conductivity, removal of PSS via $\mathrm{H}_{2} \mathrm{SO}_{4}$ has been shown to also slightly increase the Seebeck coefficient. ${ }^{[102]}$ To further optimize the power factor, post-treatment with reducing agents such as hydrazine, ${ }^{[107,115]} \mathrm{HI},{ }^{[14]}$ (dimethyl-amino) ethylene (TDAE),${ }^{[11]}$ ammonium formate $(\mathrm{AF}),{ }^{[118]}$ formic acid ${ }^{[119]}$ or bases like sodium hydroxide $(\mathrm{NaOH})^{[91,120]}$ are used to reduce charge carrier concentration, i.e. control the oxidation state of PEDOT, in combination with the previously discussed methods to increase conductivity by secondary doping.

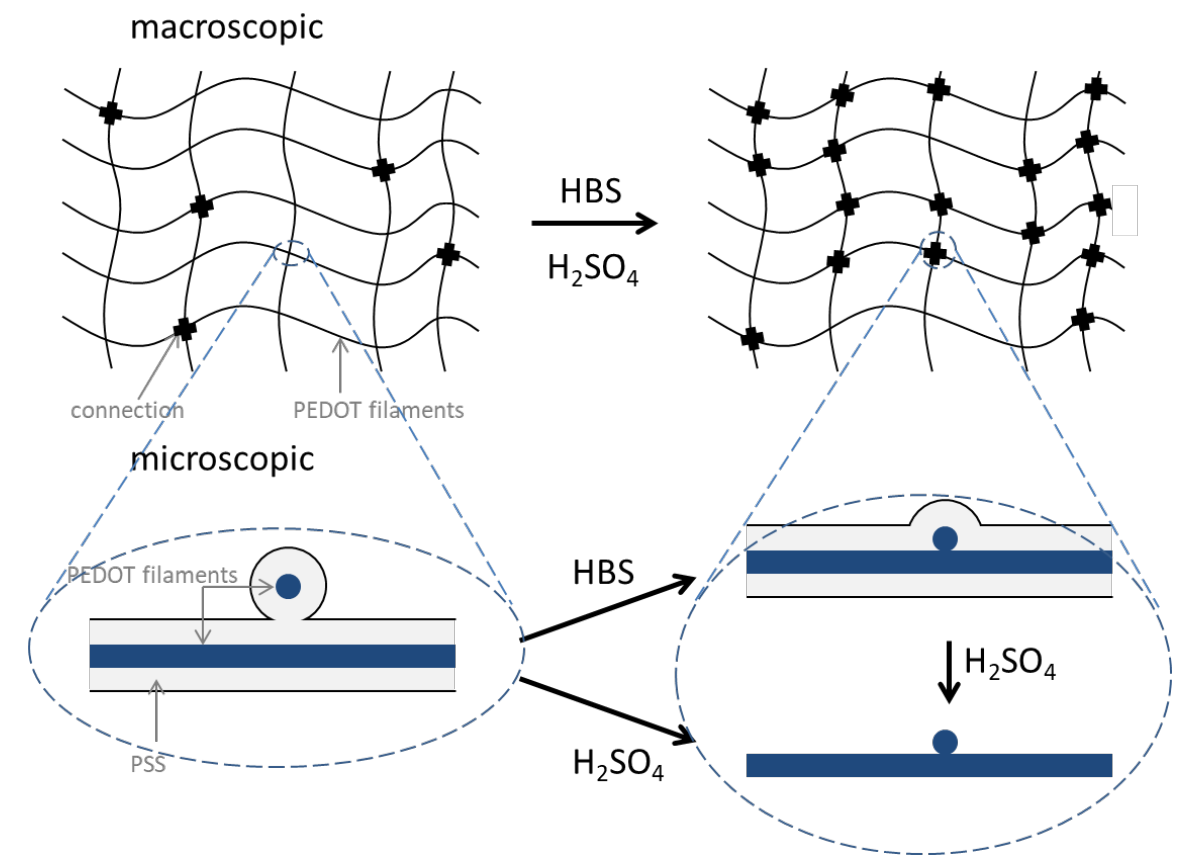

Figure 9. Schematic of the proposed mechanism for enhanced connectivity between PEDOT filaments by processing PEDOT:PSS with $\mathrm{HBS}$ or $\mathrm{H}_{2} \mathrm{SO}_{4}$. Top: macroscopic conducting network. Black crosses indicate electrical connections, enabling filaments to contribute to the network's conductivity. Bottom: proposed microscopic realizations of processing-induced electrical connection between two crossing filaments. The macroscopic conductivity can be much enhanced even if the intra-filament conductivity remains constant. Reproduced with permission. ${ }^{[102]}$ Copyright 2014, Elsevier B.V.

Summarizing these findings, it appears that high-boiling solvent and acid treatments may lead to an improved intra-filament or intra-grain transport as well as to an improved connectivity between the filaments or grains, most likely by (local) removal of the PSS between the PEDOT-rich domains, as illustrated in Figure 9. Since the inter-domain transport is the rate- 


\section{WILEY-VCH}

limiting step in the pristine material, it is likely the latter improvement that dominates the observed changes in conductivity. While the conductivity increase can amount to many orders of magnitude, the improved connectivity leaves the energetics of the charge transport largely unaffected, which is reflected in the relatively minor changes in Seebeck coefficient. Note, finally, the similarity of this explanation with the tentative mechanism proposed for the improved thermoelectric performance for blends of conjugated and insulating polymers in section 3.3 .

Finally, it should be mentioned that PEDOT:PSS is often used as a conductive matrix in a nano-composite material with carbon nano tubes or nano sheets as filler materials. ${ }^{[24-26]}$ The underlying idea is to make use of the high conductivity of the filler material and the relatively large Seebeck coefficient of the matrix. As explained in the introduction, this topic goes beyond the scope of the present report and will not be discussed further.

Table 2: Selected thermopower, electrical conductivity and power factor values for PEDOT:PSS treated in different ways, extracted from literature discussed in the text. Values are either taken from tables found in the reference materials or best estimates extracted from graphs in the reference material.

\begin{tabular}{|c|c|c|c|c|}
\hline PEDOT:PSS Treatment & $\begin{array}{l}\text { Thermopower } \\
(\mu \mathrm{V} / \mathrm{K})\end{array}$ & $\begin{array}{l}\text { Conductivity } \\
(\mathrm{S} / \mathrm{cm})\end{array}$ & $\begin{array}{l}\text { Power } \\
\text { Factor } \\
\left(\mu \mathrm{Wm}^{-1} \mathrm{~K}^{-2}\right)\end{array}$ & Ref. \\
\hline DMSO with $\mathrm{NaOH}$ dedoping & $\sim 15.8$ & $\sim 785$ & $\sim 19.6$ & [120] \\
\hline Post DMSO + Hydrazin dedoping & 49.3 & 1310 & 318.4 & [115] \\
\hline Sorbitol + TDAE dedoping & 27.47 & $\sim 295$ & $\sim 22.3$ & [111] \\
\hline $\mathrm{HI}+\mathrm{DMSO}$ (post treatment) & $\sim 30.8$ & $\sim 475$ & $\sim 45.1$ & [114] \\
\hline DMSO and Sorbitol & 11.98 & 1063 & 15.3 & [121] \\
\hline $\mathrm{H}_{2} \mathrm{SO}_{4}+\mathrm{NaOH}$ & 39.2 & 2170 & 333.5 & [91] \\
\hline Multi-layered DMSO & 10.6 & 187 & 2.1 & [122] \\
\hline Salt + DMF & 28 & 1831 & 143.6 & [108] \\
\hline $\mathrm{H}_{2} \mathrm{SO}_{4}$ & 149.47 & 65.82 & 147.1 & [123] \\
\hline$\underset{\text { treatment }}{\text { DMSO }}$ and EMBF4 post & $\sim 23.5$ & $\sim 680$ & $\sim 37.6$ & [124] \\
\hline TEMPO-OH & $\sim 22.5$ & $\sim 33$ & $\sim 1.7$ & {$[125]$} \\
\hline
\end{tabular}




\section{WILEY-VCH}

Depicted in Figure 10 is a collection of thermopower-conductivity data from literature obtained for PEDOT:PSS subjected to different treatments; for completeness also data for the PEDOT:Tos system are plotted. As noticed before, the majority of the data does not follow the empirical -1/4 power-law relationship between conductivity and thermopower observed for most doped thiophenes and other doped polymers. ${ }^{[1,55,98]}$ In line with the discussion in section 2 above, one would anticipate the correlation between conductivity and thermopower to reflect, at least partially, dopant-ion-induced changes to the DOS. ${ }^{[48]}$ However, a distinction has to be made between changes induced by secondary doping, as these are not related to any significant increase in charge carrier concentration, and changes due to PEDOT reduction or PSS removal that do lead to variations in carrier concentration. Only in the latter case are significant dopant-induced DOS changes expected to occur and could the data be expected to follow the $-1 / 4$ relationship.

The different thermopower-conductivity relation of PEDOT-type materials from other conductive polymers has been noticed before ${ }^{[48,55,126]}$ and has been successfully fitted by Kang and Snyder using the charge transport model described in section 2.1.4, see the black solid line in Figure 9. ${ }^{[55]}$ In this model PEDOT-Tos is described as having a transport parameter $s=1$, whereas all other polymers have $s=3$. According to the authors this relates to a different type of transport. ${ }^{[55]}$. 


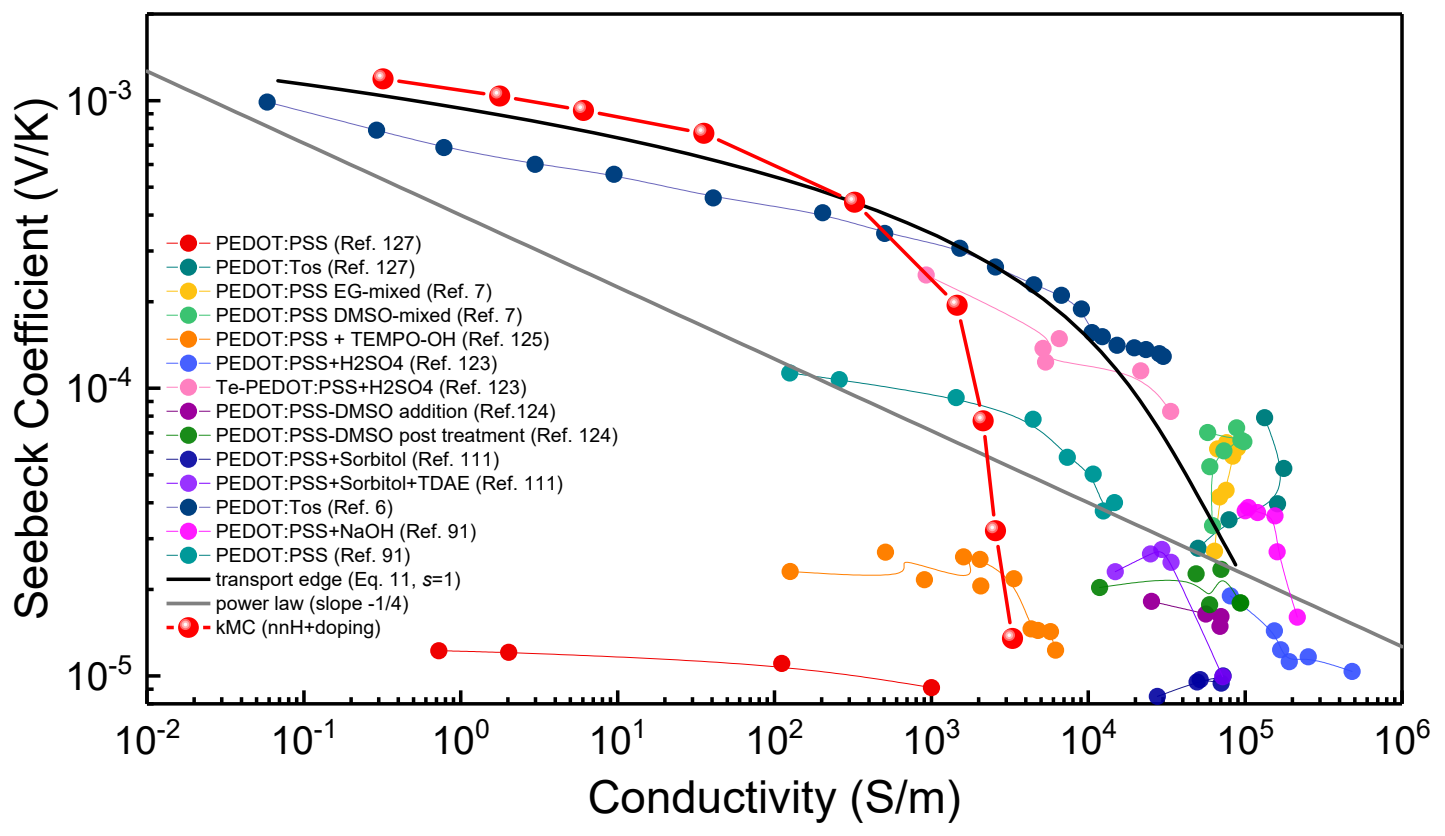

Figure 10. Collection of various thermopower-conductivity data from literature as indicated in the legend..$^{[6,7,91,111,123-125,127]}$ The grey line indicates a power law with slope -1/4, the black solid line is a fit to the transport edge model Eq. 11 (same parameters as in Figure 3c of Ref. ${ }^{[55]}, E_{F}-E_{t}=-0.3-0.3 \mathrm{eV}$ ); the red line with sphere symbols is a kMC simulation (nnH, same parameters as in Figure $2 \mathrm{c}$ above w/ rescaled conductivity prefactor and relative doping concentration $c=10^{-5}-0.43$ ).

Interestingly, straightforward application of the nearest neighbor hopping kMC model described in section 2.2 above and used for chemically doped polymers in Refs. ${ }^{[1,56]}$ and Figures 1, 2 and 4 above, roughly reproduces the curved shape of the experimental trends in Figure 10, see red line with sphere symbols. Note that the model curve can be shifted along the horizontal axis by varying the prefactor $v_{0}$ that may be different for different PEDOT systems. Like the transport edge model, it is difficult to see how this model, in which only variation in doping concentration is accounted for, can be mapped on experimental data in which morphological effects are likely to dominate over changes in doping level. As such, it is unsurprising that the agreement with experiment is largest for the PEDOT:Tos data from Ref. ${ }^{[6]}$ in which only the (de)doping level is varied. Moreover, for several PEDOT-based systems, metallic or critical behavior in the temperature-dependent conductivity is observed, which could be due to the dominance of another transport mechanism than simple 


\section{WILEY-VCH}

hopping. ${ }^{[101,127]}$ Hence, despite that phenomenological descriptions of the thermoelectric properties of PEDOT-based systems can and have been given, we feel that true, quantitative and predictive understanding is still limited and requires further attention from the community.

\subsection{Relation to $\mathrm{ZT}$}

The thermoelectric figure of merit $(\mathrm{ZT})$ is the most commonly used measure of the application potential for both $\mathrm{p}$ - and n-type OTE materials. The expression $Z T=\frac{\sigma S^{2} T}{\kappa}$ can be reformulated to relate the electrical conductivity to thermopower as: ${ }^{[55,128]}$

$S=\sqrt{\frac{\kappa \cdot Z T}{\sigma T}}$

where $\kappa$ is the thermal conductivity. In solid materials, both charge carriers and lattice vibrations can contribute to heat transport, ${ }^{[129,130]}$ i.e.

$\kappa=\kappa_{e}+\kappa_{l}$,

where $\kappa_{e}$ and $\kappa_{l}$ are the electronic and lattice vibrational contributions to thermal conductivity, respectively. In calculating the dashed lines in Figure 11, we assumed a constant $\kappa_{l} \sim 0.2 \mathrm{~W}$ $\mathrm{m}^{-1} \mathrm{~K}^{-1}$, i.e. the estimated thermal conductivity of pristine OSC. ${ }^{[2,3]}$ Instead, $\kappa_{e}$ is function of electrical conductivity, which can be described by the Wiedemann-Franz law as $\kappa_{e}=$ $\left(k_{B} / q\right)^{2} L \sigma T$, where $k_{B}$ is the Boltzmann constant, $q$ the elementary charge, and $L$ the dimensionless Lorenz factor; For simplicity, we have taken $L=L_{0}=\pi^{2} / 3$, i.e. the theoretical Sommerfeld value for a degenerate Fermi gas. ${ }^{[12]}$

To test the validity of this contour plot, reported room temperature records for both p-type (PEDOT:Tos with ZT $\sim 0.25$ (grey circle) ${ }^{[6]}$ ) and n-type (N-DMBI-doped A-DCV-DPPTT with $\mathrm{ZT} \sim 0.11$ (red circle), ${ }^{[23]}$ ) OTEs were selected from literature and plotted in Figure 10. The estimated ZT values corresponding to the positions based on their reported $S$ and $\sigma$ values are in excellent consistency with the reported ZT values. 
WILEY-VCH

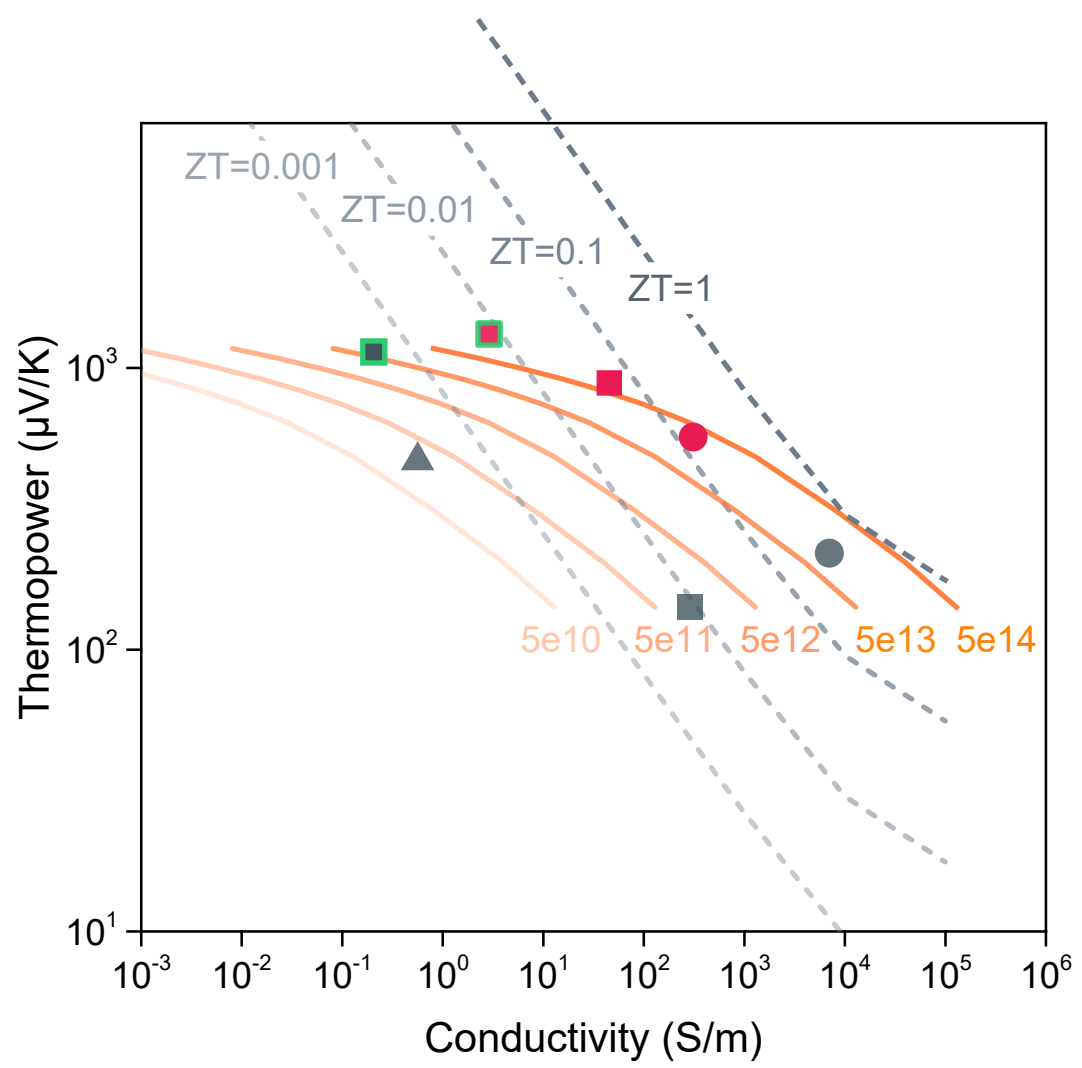

Figure 11. Contour plot of thermopower vs. conductivity parametric in the thermoelectric figure of merit ZT at $T=300 \mathrm{~K}$ from Eq. 16 (dashed lines). Solid lines are calculated from the analytical VRH model (section 2.1) with $v_{0}$ from $5 \times 10^{-10} \mathrm{~s}^{-1}$ to $5 \times 10^{-14} \mathrm{~s}^{-1}$, and other parameters: $a_{N N}=1.8 \mathrm{~nm}, \sigma_{D O S}=75 \mathrm{meV}, T=300 \mathrm{~K}, \alpha=2 \mathrm{e} 9 \mathrm{~nm}^{-1}$. Square symbols are experiments on OSCs blends and show the highest-S p-type data point from Figure 4 (gray with green edge, $\mathrm{P}_{3} \mathrm{HT}_{10}: \mathrm{PTB} 7_{90}$ ) and the highest $\mathrm{n}$-type data point from Figure 5 (red with green edge, $\mathrm{PCBM}_{01}: \mathrm{NDI}-\mathrm{CN}_{99}$ ), and the highest-PF data points from the same graphs (gray, pure $\mathrm{P} 3 \mathrm{HT}$ and red, $\mathrm{PCBM}$ ); the gray triangle marks doped pure PTB7. The red circle is for (n-type) N-DMBI-doped A-DCV-DPPTT $\left(\right.$ Ref. $^{[23]}$ ) and the gray circle is for (p-type) PEDOTTos (Ref. ${ }^{[6]}$ ). Adapted with permission. ${ }^{[64]}$ Copyright 2018, Wiley-VCH.

The relation between $S$ and $\sigma$ calculated from the VRH model as introduced in section 2.1 is also plotted in Figure 11 (orange lines), varying only the attempt-to-hop frequency. For each line the doping concentration increases towards higher electrical conductivity. The crossings of the two sets of lines indicates that, at low doping concentrations, a higher ZT for simple OSCs can be expected for higher doping concentrations. However, at larger doping concentrations, the two sets of lines run roughly parallel and other strategies are needed to further increase ZT. We plotted representative experimental results from the DOS engineering experiments discussed above (section 3) as square symbols in Figure 11, allowing a number of 


\section{WILEY-VCH}

conclusions. ${ }^{[56,64]}$ First, the p-type model system P3HT (gray square) will not likely reach relevant $\mathrm{ZT}$ values, as it, at $\mathrm{ZT} \approx 0.01$, already appears to sit close to the maximally obtainable doping concentration. ${ }^{[61]}$ Second, comparing pure PTB7 (gray triangle) with the P3HT:PTB7 blend at maximum in $S$ (gray with green edge) shows that the addition of the (P3HT) trap indeed seems to have increased ZT; a similar move starting from the grey circle would have landed near ZT $=1$. Third, although the pure P3HT (gray square) has a higher PF than the blend with PTB7 at maximum in $S$ (gray with green edge), moving along the corresponding orange line for the latter system (improving doping efficiency) would lead to higher ZT, possibly beyond $\mathrm{ZT}=0.1$. However, it is not completely evident that one can move along the orange line for blended systems, and these compounds may well be constrained to applications where high thermopower is more important than high ZT. ${ }^{[56]}$

\section{Conclusion and outlook}

In this Progress Report we have reviewed the thermoelectric properties of conjugated blends, i.e. blends in which at least one of the components is a conjugated material. In addition, we have critically reviewed several formalisms that have been used to interpret experimental results. From the experimental perspective, blending can be regarded as a valuable tool to tune materials properties in manners that are less straightforwardly achieved in single materials; examples of designing the density of states or the dielectric constant of the active material have been discussed.

Blending unavoidably brings up the joint issues of morphology control and understanding thereof. Compared to the level of understanding in e.g. organic photovoltaic blends, understanding of the role of morphology on thermoelectric properties of organic materials, and especially blends thereof, is very limited. This is especially true for the thermal conductivity that, also in the broader field of OSC, is largely unexplored. As such, inclusion of morphology in analytical or numerical models for charge, heat and energy transport will be 


\section{WILEY-VCH}

an important and urgent step towards a comprehensive model that can rationalize the properties of organic thermoelectrics and guide their design.

Irrespective of the presence or absence of quantitative models, the results obtained so far point to the need to process multi-component materials in such a way that the long-range connectivity of the charge transporting phase(s) is optimized. While e.g. physical interruptions in conducting filaments do not much affect the energetics of charge transport and therefore the thermopower, they seriously hamper conductivity. General processing strategies to achieve long-range connectivity do not seem to have been reported - if they even exist. In absence of good morphology control in multicomponent blends, and judging results reported so far, it appears unlikely that materials reported so far are already near a fundamental upper limit and one may expect further performance increases.

Seen from a broader perspective, conjugated thermoelectric blends with promising properties, based on interesting and novel concepts, have been demonstrated. Nevertheless, materials with figures of merit ZT that are relevant beyond extreme niche applications are still to be reported. It remains to be proven that concepts that can be used to improve a mediocre thermoelectric can also be used to improve the current state-of-the-art to reach, e.g., ZT $\geq 1$.

\section{Acknowledgements}

The research by G.Z. was supported by the China Scholarship Council (CSC). H.A gratefully acknowledges funding by the Knut och Alice Wallenbergs Stiftelse (project "Tail of the Sun"). 


\section{WILEY-VCH}

References

[1] N. Dubey, M. Leclerc, J. Polym. Sci. Part B Polym. Phys. 2011, 49, 467.

[2] D. Venkateshvaran, M. Nikolka, A. Sadhanala, V. Lemaur, M. Zelazny, M. Kepa, M. Hurhangee, A. J. Kronemeijer, V. Pecunia, I. Nasrallah, I. Romanov, K. Broch, I.

McCulloch, D. Emin, Y. Olivier, J. Cornil, D. Beljonne, H. Sirringhaus, Nature 2014, 515,384 .

[3] F. Zhang, Y. Zang, D. Huang, C. Di, X. Gao, H. Sirringhaus, D. Zhu, Adv. Funct. Mater. 2015, 25, 3004.

[4] C. J. Glassbrenner, G. A. Slack, Phys. Rev. 1964, 134, A1058.

[5] T. M. Tritt, M. A. Subramanian, MRS Bull. 2006, 31, 188.

[6] O. Bubnova, Z. U. Khan, A. Malti, S. Braun, M. Fahlman, M. Berggren, X. Crispin, Nat. Mater. 2011, 10, 429.

[7] G.-H. Kim, L. Shao, K. Zhang, K. P. Pipe, Nat. Mater. 2013, 12, 719.

[8] S. van van Reenen, M. Kemerink, Org. Electron. 2014, 15, 2250.

[9] G.-H. Kim, J. Kim, K. P. Pipe, Appl. Phys. Lett. 2016, 108, 093301.

[10] A. Shakouri, Annu. Rev. Mater. Res. 2011, 41, 399.

[11] A. M. Glaudell, J. E. Cochran, S. N. Patel, M. L. Chabinyc, Adv. Energy Mater. 2015, 5, 1401072.

[12] A. Weathers, Z. U. Khan, R. Brooke, D. Evans, M. T. Pettes, J. W. Andreasen, X. Crispin, L. Shi, Adv. Mater. 2015, 27, 2101.

[13] S. N. Patel, A. M. Glaudell, K. A. Peterson, E. M. Thomas, K. A. O’Hara, E. Lim, M. L. Chabinyc, Sci. Adv. 2017, 3, e1700434.

[14] J. Hynynen, D. Kiefer, L. Yu, R. Kroon, R. Munir, A. Amassian, M. Kemerink, C. Müller, Macromolecules 2017, 50, 8140.

[15] J. Hynynen, D. Kiefer, C. Müller, RSC Adv. 2018, 8, 1593.

[16] K. Kang, S. Watanabe, K. Broch, A. Sepe, A. Brown, I. Nasrallah, M. Nikolka, Z. Fei, M. Heeney, D. Matsumoto, K. Marumoto, H. Tanaka, S. Kuroda, H. Sirringhaus, Nat. Mater. 2016, DOI 10.1038/nmat4634.

[17] D. Kiefer, L. Yu, E. Fransson, A. Gómez, D. Primetzhofer, A. Amassian, M. CampoyQuiles, C. Müller, $A d v$. Sci. 2017, 4, n/a.

[18] J. Liu, L. Qiu, G. Portale, M. Koopmans, G. ten Brink, J. C. Hummelen, L. J. A. Koster, Adv. Mater. 2017, 29, 1701641.

[19] J. Liu, L. Qiu, R. Alessandri, X. Qiu, G. Portale, J. Dong, W. Talsma, G. Ye, A. A. Sengrian, P. C. T. Souza, M. A. Loi, R. C. Chiechi, S. J. Marrink, J. C. Hummelen, L. J. A. Koster, Adv. Mater. 2018, 1704630.

[20] C.-K. Mai, R. A. Schlitz, G. M. Su, D. Spitzer, X. Wang, S. L. Fronk, D. G. Cahill, M. L. Chabinyc, G. C. Bazan, J. Am. Chem. Soc. 2014, 136, 13478.

[21] B. Russ, M. J. Robb, F. G. Brunetti, P. L. Miller, E. E. Perry, S. N. Patel, V. Ho, W. B. Chang, J. J. Urban, M. L. Chabinyc, C. J. Hawker, R. A. Segalman, Adv. Mater. 2014, 26,3473 .

[22] K. Shi, F. Zhang, C.-A. Di, T.-W. Yan, Y. Zou, X. Zhou, D. Zhu, J.-Y. Wang, J. Pei, J. Am. Chem. Soc. 2015, 137, 6979.

[23] D. Huang, H. Yao, Y. Cui, Y. Zou, F. Zhang, C. Wang, H. Shen, W. Jin, J. Zhu, Y. Diao, W. Xu, C. Di, D. Zhu, J. Am. Chem. Soc. 2017, 139, 13013.

[24] B. T. McGrail, A. Sehirlioglu, E. Pentzer, Angew. Chem. Int. Ed. 2015, 54, 1710.

[25] Y. Xue, C. Gao, L. Liang, X. Wang, G. Chen, J. Mater. Chem. A 2018, 6, 22381.

[26] Blackburn Jeffrey L., Ferguson Andrew J., Cho Chungyeon, Grunlan Jaime C., $A d v$. Mater. 2018, 30, 1704386.

[27] M. Silver, L. Pautmeier, H. Bässler, Solid State Commun. 1989, 72, 177.

[28] V. I. Arkhipov, P. Heremans, E. V. Emelianova, H. Bässler, Phys. Rev. B 2005, 71, 045214. 


\section{WILEY-VCH}

[29] V. I. Arkhipov, E. V. Emelianova, P. Heremans, H. Bässler, Phys. Rev. B 2005, 72, 235202.

[30] A. Mityashin, Y. Olivier, T. Van Regemorter, C. Rolin, S. Verlaak, N. G. Martinelli, D. Beljonne, J. Cornil, J. Genoe, P. Heremans, Adv. Mater. 2012, 24, 1535.

[31] G. Zuo, H. Abdalla, M. Kemerink, Phys. Rev. B 2016, 93, 235203.

[32] P. Pingel, R. Schwarzl, D. Neher, Appl. Phys. Lett. 2012, 100, 143303.

[33] P. Pingel, D. Neher, Phys. Rev. B 2013, 87, 115209.

[34] G. Zuo, Z. Li, O. Andersson, H. Abdalla, E. Wang, M. Kemerink, J. Phys. Chem. C 2017, 121, 7767.

[35] M. L. Tietze, J. Benduhn, P. Pahner, B. Nell, M. Schwarze, H. Kleemann, M. Krammer, K. Zojer, K. Vandewal, K. Leo, Nat. Commun. 2018, 9, 1182.

[36] D. Di Nuzzo, C. Fontanesi, R. Jones, S. Allard, I. Dumsch, U. Scherf, E. von Hauff, S. Schumacher, E. Da Como, Nat. Commun. 2015, 6, DOI 10.1038/ncomms7460.

[37] H. Méndez, G. Heimel, S. Winkler, J. Frisch, A. Opitz, K. Sauer, B. Wegner, M. Oehzelt, C. Röthel, S. Duhm, D. Többens, N. Koch, I. Salzmann, Nat. Commun. 2015, 6, 8560.

[38] I. Salzmann, G. Heimel, M. Oehzelt, S. Winkler, N. Koch, Acc. Chem. Res. 2016, 49, 370.

[39] A. Miller, E. Abrahams, Phys. Rev. 1960, 120, 745.

[40] H. C. F. Martens, I. N. Hulea, I. Romijn, H. B. Brom, W. F. Pasveer, M. A. J. Michels, Phys. Rev. B 2003, 67, DOI 10.1103/PhysRevB.67.121203.

[41] R. Coehoorn, W. F. Pasveer, P. A. Bobbert, M. A. J. Michels, Phys. Rev. B 2005, 72, 155206.

[42] S. D. Baranovskii, Phys. Status Solidi B 2014, 251, 487.

[43] H. Bässler, Phys. Status Solidi B 1993, 175, 15.

[44] N. Tessler, Y. Preezant, N. Rappaport, Y. Roichman, Adv. Mater. 2009, 21, 2741.

[45] A. V. Nenashev, J. O. Oelerich, S. D. Baranovskii, J. Phys. Condens. Matter 2015, 27, 093201.

[46] J. Cottaar, L. J. A. Koster, R. Coehoorn, P. A. Bobbert, Phys. Rev. Lett. 2011, 107, 136601.

[47] V. I. Arkhipov, P. Heremans, E. V. Emelianova, G. J. Adriaenssens, H. Bässler, J. Phys. Condens. Matter 2002, 14, 9899.

[48] H. Abdalla, G. Zuo, M. Kemerink, Phys. Rev. B 2017, 96, 241202.

[49] G. Zuo, H. Abdalla, M. Kemerink, Phys. Rev. B 2018, 97, 079902.

[50] C. Gaul, S. Hutsch, M. Schwarze, K. S. Schellhammer, F. Bussolotti, S. Kera, G. Cuniberti, K. Leo, F. Ortmann, Nat. Mater. 2018, 17, 439.

[51] H. Fritzsche, Solid State Commun. 1971, 9, 1813.

[52] S. D. Baranovskii, T. Faber, F. Hensel, P. Thomas, J. Phys. Condens. Matter 1997, 9, 2699.

[53] R. Schmechel, J. Appl. Phys. 2003, 93, 4653.

[54] S. Ihnatsenka, X. Crispin, I. V. Zozoulenko, Phys. Rev. B 2015, 92, 035201.

[55] S. D. Kang, G. J. Snyder, Nat. Mater. 2017, 16, 252.

[56] G. Zuo, X. Liu, M. Fahlman, M. Kemerink, Adv. Funct. Mater. n.d., n/a.

[57] J. Cottaar, L. J. A. Koster, R. Coehoorn, P. A. Bobbert, Phys. Rev. Lett. 2011, 107, 136601.

[58] D. T. Scholes, S. A. Hawks, P. Y. Yee, H. Wu, J. R. Lindemuth, S. H. Tolbert, B. J. Schwartz, J. Phys. Chem. Lett. 2015, 6, 4786.

[59] G. D. Mahan, J. O. Sofo, Proc. Natl. Acad. Sci. 1996, 93, 7436.

[60] J. Sun, M.-L. Yeh, B. J. Jung, B. Zhang, J. Feser, A. Majumdar, H. E. Katz, Macromolecules 2010, 43, 2897.

[61] G. Zuo, O. Andersson, H. Abdalla, M. Kemerink, Appl. Phys. Lett. 2018, 112, 083303. 


\section{WILEY-VCH}

[62] D. T. Scholes, S. A. Hawks, P. Y. Yee, H. Wu, J. R. Lindemuth, S. H. Tolbert, B. J. Schwartz, J. Phys. Chem. Lett. 2015, 6, 4786.

[63] Q. Bao, X. Liu, S. Braun, F. Gao, M. Fahlman, Adv. Mater. Interfaces 2015, 2, n/a.

[64] G. Zuo, Z. Li, E. Wang, M. Kemerink, Adv. Electron. Mater. 2018, 4, 1700501.

[65] R. Kroon, D. A. Mengistie, D. Kiefer, J. Hynynen, J. D. Ryan, L. Yu, C. Müller, Chem. Soc. Rev. 2016, 45, 6147.

[66] G. Zuo, X. Liu, M. Fahlman, M. Kemerink, ACS Appl. Mater. Interfaces 2018, 10, 9638.

[67] J. K. Lee, W. L. Ma, C. J. Brabec, J. Yuen, J. S. Moon, J. Y. Kim, K. Lee, G. C. Bazan, A. J. Heeger, J. Am. Chem. Soc. 2008, 130, 3619.

[68] Y. Liu, J. Zhao, Z. Li, C. Mu, W. Ma, H. Hu, K. Jiang, H. Lin, H. Ade, H. Yan, Nat. Commun. 2014, 5, ncomms6293.

[69] E. Moons, J. Phys. Condens. Matter 2002, 14, 12235.

[70] Y. Shi, J. Liu, Y. Yang, J. Appl. Phys. 2000, 87, 4254.

[71] H. N. Tsao, D. Cho, J. W. Andreasen, A. Rouhanipour, D. W. Breiby, W. Pisula, K. Müllen, Adv. Mater. 2009, 21, 209.

[72] S. H. Park, A. Roy, S. Beaupré, S. Cho, N. Coates, J. S. Moon, D. Moses, M. Leclerc, K. Lee, A. J. Heeger, Nat. Photonics 2009, 3, nphoton.2009.69.

[73] S. Hwang, W. J. Potscavage, R. Nakamichi, C. Adachi, Org. Electron. 2016, 31, 31.

[74] A. Hamidi-Sakr, L. Biniek, J.-L. Bantignies, D. Maurin, L. Herrmann, N. Leclerc, P. Lévêque, V. Vijayakumar, N. Zimmermann, M. Brinkmann, Adv. Funct. Mater. 2017, $27, \mathrm{n} / \mathrm{a}$.

[75] J. Zhao, Y. Li, G. Yang, K. Jiang, H. Lin, H. Ade, W. Ma, H. Yan, Nat. Energy 2016, 1, 15027.

[76] Y. Sun, G. C. Welch, W. L. Leong, C. J. Takacs, G. C. Bazan, A. J. Heeger, Nat. Mater. 2012, 11, 44.

[77] S. J. Lou, J. M. Szarko, T. Xu, L. Yu, T. J. Marks, L. X. Chen, J. Am. Chem. Soc. 2011, 133, 20661.

[78] Y. Liu, J. Zhao, Z. Li, C. Mu, W. Ma, H. Hu, K. Jiang, H. Lin, H. Ade, H. Yan, Nat. Commun. 2014, 5, 5293.

[79] H.-C. Liao, C.-C. Ho, C.-Y. Chang, M.-H. Jao, S. B. Darling, W.-F. Su, Mater. Today 2013, 16, 326.

[80] P.-W. Liang, C.-Y. Liao, C.-C. Chueh, F. Zuo, S. T. Williams, X.-K. Xin, J. Lin, A. K.Y. Jen, Adv. Mater. 2014, 26, 3748.

[81] V. Ambegaokar, B. I. Halperin, J. S. Langer, Phys. Rev. B 1971, 4, 2612.

[82] P. Peumans, S. Uchida, S. R. Forrest, Nature 2003, 425, nature01949.

[83] R. A. Marsh, C. Groves, N. C. Greenham, J. Appl. Phys. 2007, 101, 083509.

[84] C. O. Yoon, M. Reghu, D. Moses, A. J. Heeger, Y. Cao, Synth. Met. 1994, 63, 47.

[85] C. O. Yoon, M. Reghu, D. Moses, Y. Cao, A. J. Heeger, Synth. Met. 1995, 69, 255.

[86] V. Jousseaume, M. Morsli, A. Bonnet, O. Tesson, S. Lefrant, J. Appl. Polym. Sci. 1998, 67, 1205.

[87] C. K. Subramaniam, A. B. Kaiser, P. W. Gilberd, C.-J. Liu, B. Wessling, Solid State Commun. 1996, 97, 235.

[88] Y. Wang, J. Zhou, R. Yang, J. Phys. Chem. C 2011, 115, 24418.

[89] G. Lu, L. Bu, S. Li, X. Yang, Adv. Mater. 2014, 26, 2359.

[90] D. Kiefer, L. Yu, E. Fransson, A. Gómez, D. Primetzhofer, A. Amassian, M. CampoyQuiles, C. Müller, Adv. Sci. 2017, 4, 1600203.

[91] Z. Fan, P. Li, D. Du, J. Ouyang, Adv. Energy Mater. 2017, 7, 1602116.

[92] C. Y. Yang, Y. Cao, P. Smith, A. J. Heeger, Synth. Met. 1993, 53, 293.

[93] G. Lu, H. Tang, Y. Huan, S. Li, L. Li, Y. Wang, X. Yang, Adv. Funct. Mater. 2010, 20, 1714. 


\section{WILEY-VCH}

[94] H. Yao, Z. Fan, H. Cheng, X. Guan, C. Wang, K. Sun, J. Ouyang, Macromol. Rapid Commun. 2018, 39, 1700727.

[95] C. Yu, Y. S. Kim, D. Kim, J. C. Grunlan, Nano Lett. 2008, 8, 4428.

[96] C. Meng, C. Liu, S. Fan, Adv. Mater. 2010, 22, 535.

[97] N. E. Coates, S. K. Yee, B. McCulloch, K. C. See, A. Majumdar, R. A. Segalman, J. J. Urban, Adv. Mater. 2013, 25, 1629.

[98] A. B. Kaiser, Rep. Prog. Phys. 2001, 64, 1.

[99] H. Wang, U. Ail, R. Gabrielsson, M. Berggren, X. Crispin, Adv. Energy Mater. 2015, 5, n/a.

[100] U. Ail, M. J. Jafari, H. Wang, T. Ederth, M. Berggren, X. Crispin, Adv. Funct. Mater. 2016, 26, 6288.

[101] K. van de Ruit, R. I. Cohen, D. Bollen, T. van Mol, R. Yerushalmi-Rozen, R. A. J. Janssen, M. Kemerink, Adv. Funct. Mater. 2013, 23, 5778.

[102] S. van Reenen, M. Scheepers, K. van de Ruit, D. Bollen, M. Kemerink, Org. Electron. 2014, 15, 3710 .

[103] A. M. Nardes, M. Kemerink, R. A. J. Janssen, J. A. M. Bastiaansen, N. M. M. Kiggen, B. M. W. Langeveld, A. J. J. M. van Breemen, M. M. de Kok, Adv. Mater. 2007, 19, 1196.

[104] A. Nardes, M. Kemerink, R. Janssen, Phys. Rev. B 2007, 76, 085208.

[105] S. Ihnatsenka, Phys. Rev. B 2016, 94, 195202.

[106] J. Y. Kim, J. H. Jung, D. E. Lee, J. Joo, Synth. Met. 2002, 126, 311.

[107] H. Park, S. H. Lee, F. S. Kim, H. H. Choi, I. W. Cheong, J. H. Kim, J. Mater. Chem. A 2014, 2, 6532.

[108] S. Zhang, Z. Fan, X. Wang, Z. Zhang, J. Ouyang, J. Mater. Chem. A 2018, 6, 7080.

[109] S. K. M. Jönsson, J. Birgerson, X. Crispin, G. Greczynski, W. Osikowicz, A. W.

Denier van der Gon, W. R. Salaneck, M. Fahlman, Synth. Met. 2003, 139, 1.

[110] A. M. Nardes, R. A. J. Janssen, M. Kemerink, Adv. Funct. Mater. 2008, 18, 865.

[111] E. Yang, J. Kim, B. J. Jung, J. Kwak, J. Mater. Sci. Mater. Electron. 2015, 26, 2838.

[112] Q. Wei, M. Mukaida, Y. Naitoh, T. Ishida, Adv. Mater. 2013, 25, 2831.

[113] A. Aleshin, R. Kiebooms, R. Menon, A. J. Heeger, Synth. Met. 1997, 90, 61.

[114] L. Zhang, H. Deng, S. Liu, Q. Zhang, F. Chen, Q. Fu, RSC Adv. 2015, 5, 105592.

[115] S. H. Lee, H. Park, S. Kim, W. Son, I. W. Cheong, J. H. Kim, J. Mater. Chem. A 2014, 2, 7288 .

[116] Adv. Funct. Mater. 2013, 23, 5778.

[117] Y. Xia, K. Sun, J. Ouyang, Adv. Mater. 2012, 24, 2436.

[118] T.-C. Tsai, H.-C. Chang, C.-H. Chen, W.-T. Whang, Org. Electron. 2011, 12, 2159.

[119] D. A. Mengistie, C.-H. Chen, K. M. Boopathi, F. W. Pranoto, L.-J. Li, C.-W. Chu, ACS Appl. Mater. Interfaces 2015, 7, 94.

[120] T.-C. Tsai, H.-C. Chang, C.-H. Chen, Y.-C. Huang, W.-T. Whang, Org. Electron. 2014, $15,641$.

[121] H. J. Oh, J. G. Jang, J.-G. Kim, J.-I. Hong, J. Kim, J. Kwak, S. H. Kim, S. Shin, Sci. Rep. 2017, 7, 13287.

[122] V. Andrei, K. Bethke, F. Madzharova, S. Beeg, A. Knop-Gericke, J. Kneipp, K.

Rademann, Adv. Electron. Mater. 2017, 3, 1600473.

[123] E. Jin Bae, Y. Hun Kang, K.-S. Jang, S. Yun Cho, Sci. Rep. 2016, 6, 18805.

[124] J. Luo, D. Billep, T. Waechtler, T. Otto, M. Toader, O. Gordan, E. Sheremet, J. Martin, M. Hietschold, D. R. T. Zahn, T. Gessner, J. Mater. Chem. A 2013, 1, 7576.

[125] E. P. Tomlinson, M. J. Willmore, X. Zhu, S. W. A. Hilsmier, B. W. Boudouris, ACS Appl. Mater. Interfaces 2015, 7, 18195.

[126] B. Russ, A. Glaudell, J. J. Urban, M. L. Chabinyc, R. A. Segalman, Nat. Rev. Mater. 2016, $1,16050$. 


\section{WILEY-VCH}

[127] O. Bubnova, Z. U. Khan, H. Wang, S. Braun, D. R. Evans, M. Fabretto, P. HojatiTalemi, D. Dagnelund, J.-B. Arlin, Y. H. Geerts, S. Desbief, D. W. Breiby, J. W. Andreasen, R. Lazzaroni, W. M. Chen, I. Zozoulenko, M. Fahlman, P. J. Murphy, M. Berggren, X. Crispin, Nat. Mater. 2014, 13, 190.

[128] G. Zuo, Z. Li, E. Wang, M. Kemerink, Adv. Electron. Mater. 2017, 4, 1700501.

[129] G. S. Kumar, J. W. Vandersande, T. Klitsner, R. O. Pohl, G. A. Slack, Phys. Rev. B $1985,31,2157$.

[130] D G Cahill, and R. O. Pohl, Annu. Rev. Phys. Chem. 1988, 39, 93.

((Insert Figure here. Note: Please do not combine figure and caption in a textbox or frame.))

Figure 1. ((Figure Caption.))

Reproduced with permission. ${ }^{[\text {Ref.] }}$ Copyright Year, Publisher. ((delete if not applicable))

Table 1. ((Table Caption. Note: Please do not combine table and caption in a textbox or frame and do not submit tables as graphics, please use Word's "insert table" function.))

\begin{tabular}{|c|c|c|c|c|}
\hline $\begin{array}{l}\text { Head } 1 \\
\text { [units] }^{\mathrm{a})}\end{array}$ & Head 2 & Head 3 & Head 4 & $\begin{array}{c}\text { Head } 5 \\
\text { [units] }\end{array}$ \\
\hline Column 1 & Column 2 & Column $3^{\text {b) }}$ & Column 4 & Column 5 \\
\hline Column 1 & Column 2 & Column 3 & Column 4 & Column 5 \\
\hline
\end{tabular}




\section{WILEY-VCH}

\section{Author Biographies}

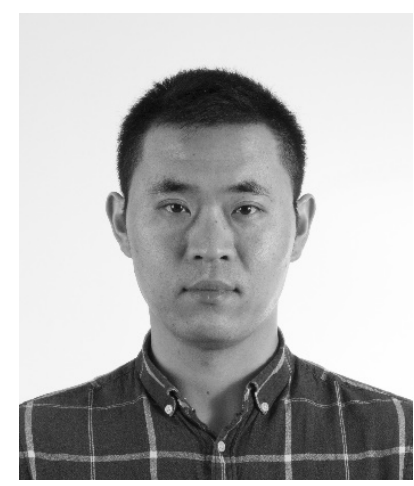

Guangzheng Zuo received his Master's degree at Nanjing University of Posts and Telecommunications under the supervision of Professor Wei Wei in 2014, and his PhD degree at Linköping University under the supervision of Professor Martijn Kemerink in 2018. His current research interests focus on charge transport in organic electronic materials, especially in the field of organic solar cells and organic thermoelectrics.

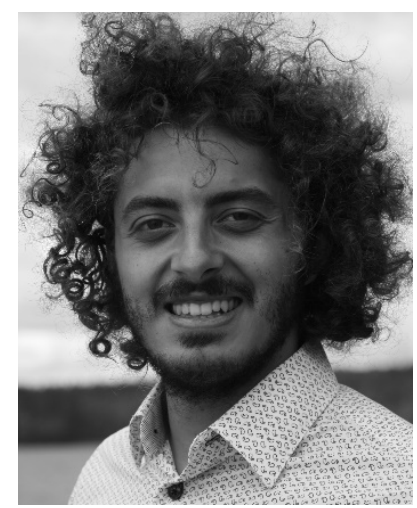

Hassan Abdalla undertook research of carrier lifetime improvement of wide band gap semiconductors as part of the Master's education at Linköping University, Sweden in 2012. Moving on to organic semiconductors for his $\mathrm{PhD}$ education, he studied their charge and energy transport by a combining numerical and experimental techniques under the supervision of Professor Martijn Kemerink, obtaining his $\mathrm{PhD}$ degree in 2018. Presently he works as consultant for the semiconductor industry.

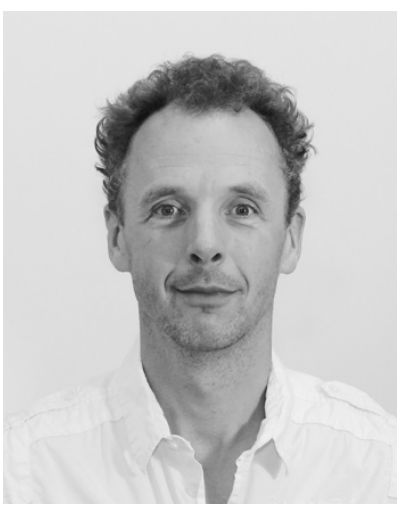

Martijn Kemerink is group leader and full professor in Applied Physics at Linköping University since 2014. He obtained his PhD in Applied Physics from Eindhoven University of Technology (1998) on many body effects in III/V semiconductor heterostructures. Later, he shifted his research focus towards scanning probe microscopy and organic electronics. His current research focuses on the physics of organic materials and devices for energy harvesting and memory applications. 


\section{WILEY-VCH}

Strategies to increase thermoelectric performance of doped organic semiconductors by blending are reviewed. Experimental results are, where possible, compared to analytical and numerical models. Several promising strategies to increase conductivity and/or thermopower are experimentally identified in recent literature. In contrast, formal understanding, especially of the role of morphology, is somewhat lagging behind.

\section{Keyword: Thermoelectric blends}

Guangzheng Zuo, Hassan Abdalla and Martijn Kemerink*

Title: Conjugated Polymer Blends for Organic Thermoelectrics

ToC figure:

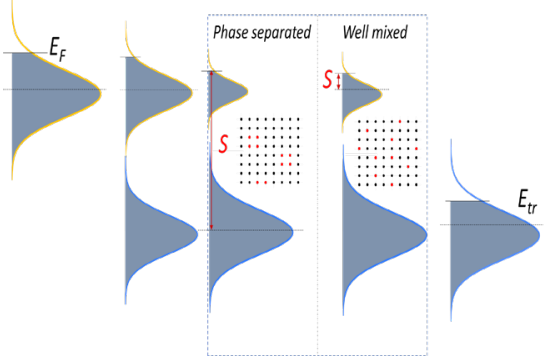

